\title{
Efficient Scheduling and Power Allocation for D2D-assisted Wireless Caching Networks
}

\author{
Lin Zhang, Student Member, IEEE, Ming Xiao, Senior Member, IEEE, \\ Gang Wu, Member, IEEE, and Shaoqian Li, Fellow, IEEE
}

\begin{abstract}
We study an one-hop device-to-device (D2D) assisted wireless caching network, where popular files are randomly and independently cached in the memory of end-users. Each user may obtain the requested files from its own memory without any transmission, or from a helper through an onehop D2D transmission, or from the base station (BS). We formulate a joint D2D link scheduling and power allocation problem to maximize the system throughput. However, the problem is non-convex and obtaining an optimal solution is computationally hard. Alternatively, we decompose the problem into a D2D link scheduling problem and an optimal power allocation problem. To solve the two subproblems, we first develop a D2D link scheduling algorithm to select the largest number of D2D links satisfying both the signal to interference plus noise ratio (SINR) and the transmit power constraints. Then, we develop an optimal power allocation algorithm to maximize the minimum transmission rate of the scheduled D2D links. Numerical results indicate that both the number of the scheduled D2D links and the system throughput can be improved simultaneously with the Zipf-distribution caching scheme, the proposed D2D link scheduling algorithm, and the proposed optimal power allocation algorithm compared with the state of arts.
\end{abstract}

\section{Index Terms}

D2D transmission, link scheduling, power allocation, wireless caching.

Lin Zhang, Gang Wu, and Shaoqian Li are with the National Key Lab of Science and Technology on Communications, University of Electronic Science and Technology of China, Chengdu, China, emails: linzhang1913@gmail.com, \{wugang99, lsq\}@uestc.edu.cn; Ming Xiao is with the School of Electrical Engineering, Royal Institute of Technology, KTH, email: mxiao@ieee.org. 


\section{INTRODUCTION}

With the emergence of various applications, especially multimedia, mobile data has been explosively increasing in recent years. It is predicted that the mobile traffic will grow by more than 200 to 2000 times in the next few years according to a study from IMT 2020 [1]. The huge data amount pushes operators to provide high-throughput wireless access services. However, the current wireless access technology is already quite close to the performance limits and thus new communication strategies are needed to meet the increasing requirement from mobile subscribers.

One promising approach is to use heterogeneous networks [2], [3], where one cell is divided into multiple small cells, i.e., microcells, picocells, and femtocells. Within each small cell, one low-power base station (LPBS) is equipped to serve the users in coverage. Specially, each LPBS is connected to the base station (BS) of the cell with a backhaul, e.g., high-speed fiber [4]. Then, the requested files by a user are first transmitted from the BS to the LPBS through the backhaul and then transmitted from the LPBS to the user. The deployment of LPBS shortens the wireless transmission distance and results in a spatial gain, which boosts the system throughput dramatically compared with the conventional cellular networks. However, it may be very expensive to establish and maintain the LPBS as well as the backhaul. This hinders the application of the heterogeneous networks in practice.

Alternatively, wireless caching recently has attracted a lot of attentions for the advantages of fast response and without heavily relying on the backhaul [5], [6]. As observed that some files, e.g., video clips, usually remain popular in a certain period of time, say one day, one week, even one month. Meanwhile, with the fast development of integrated circuit (IC) technologies, the price of storage memory drops quickly. Then, the storage capacity in devices can be utilized for wireless caching. Specifically, some popular files can be cached into the memory of users during the off-load time, say middle night, such that users may obtain the requested files from the devices rather than the BS in the peak-load time, say daytime. Notably, a popular file may be requested by multiple users. Thus, more user requests can be accommodated with the help of wireless caching during the peak-load time. This increases the system throughput significantly. Besides, high-speed backhauls are not required for the wireless caching since the cached files can be transmitted through wireless channels during the off-load time.

In this paper, we focus on the wireless caching networks with one-hop device-to-device (D2D) 
communication among users, since D2D communication has been shown to be a promising candidate to improve the system throughput [7]. Then, some popular files are cached into the memory of users and each user may obtain the requested files from its own memory without any transmission, or from a helper through an one-hop D2D transmission, or from the BS. We formulate a joint D2D link scheduling and power allocation problem to maximize the system throughput. However, the problem is non-convex and obtaining an optimal solution is computationally hard. Alternatively, we seek to obtain a suboptimal solution with reasonable complexity. Briefly, we intend to schedule the D2D links with strong communication channels and weak interference channels and allocate the power to the scheduled D2D links fairly. Thus, we decompose the joint optimization problem into a D2D link scheduling problem and an optimal power allocation problem. To solve the two subproblems, we first develop a D2D link scheduling algorithm to select the largest number of D2D links satisfying both the signal to interference plus noise ratio (SINR) and the transmit power constraints. Then, we develop an optimal power allocation algorithm to maximize the minimum transmission rate of the scheduled D2D links. Numerical results indicate that both the number of the scheduled D2D links and the system throughput can be improved simultaneously with the Zipf-distribution caching scheme, the proposed D2D link scheduling algorithm, and the proposed optimal power allocation algorithm compared with the state of arts.

Related literature: Wireless caching has recently been studied from theoretical perspectives in [8]-[13]. Specifically, [8] and [9] study a typical server-user model, where a file server transmits data bits on a shared link (broadcasting channel) to satisfy the request of each user. The objective is to minimize the transmission rate on the shared link. Then, [8] and [9] propose centralized and decentralized caching schemes to exploit both local and global caching gains, and thus achieve multiplicative peak rate reduction on the shared link. [10] proposes an online coded caching scheme and achieves the order-optimal long-term performance on the shared link. Besides the single-layer caching in [8]-[10], [11] proposes an order-optimal file placement and delivery scheme for a two-layer wireless caching network. [12] generalizes the result in [11] and studies the multi-requests wireless caching networks, where each user requests more than one files. Then, [12] proposes a caching scheme based on multiple groupcast index coding and achieves the order-optimal performance. [13] extends the result in [12] and provides the complete orderoptimal characterization of the transmission rate on the shard link. 
Considering the popularity diversity of files, [14]-[17] study nonuniform coded caching networks, where files are assumed to have different popularity. [14] considers a wireless caching network with one helper and multiple users, and develops an order-optimal coded caching scheme. [15] generalizes the result in [14] to multiple helpers and users, and optimizes the trade-off among the peak rate on the shared link, the memory size in helpers, and the access cost of users. [16] divides the popularity of files into several discrete levels and derives an information-theoretic outer bound for the nonuniform network. Different from [16], where the peak rate on the shared link is considered, [17] optimizes the long-term performance. That is, [17] considers the average rate on the shared link and develops simple order-optimal schemes. Furthermore, [18]-[22] study more practical scenarios and design effective caching schemes subject to heterogenous cache sizes, delivery delay constraint, security problem, pricing problem, and streaming schedule problem.

To further improve the system performance, D2D communication is proposed for wireless caching [23]-[28]. [23] considers the D2D communication in wireless caching networks from the perspective of information theory and proposes a deterministic caching scheme and a random caching scheme, both of which may achieve the information theoretic outer bound within a constant multiplicative factor. Then, [24] and [25] provide the basic principle and system performance of a wireless caching network with D2D communication, and show that the gain from the unicast D2D communication is comparable to the gain from the coded BS multicast. [26] proposes a novel architecture to increase the system throughput and obtains the optimal collaboration distance of D2D communication. Different from [23]-[26], where one-hop D2D communication is allowed, [27] and [28] consider multi-hop D2D wireless caching networks, where a requested file can be directly obtained through an one-hop D2D transmission or indirectly obtained after multi-hop D2D transmissions. More specifically, [27] and [28] study the throughput scaling law and propose a decentralized caching scheme and a multi-hop D2D transmission scheme. With the schemes, the optimal throughput scaling law is achieved and outperforms the scaling law in one-hop D2D communication networks.

Contributions: We adopt the architecture similar to [26], where each user may obtain the requested files from its own memory without any transmission, or from a helper through an onehop D2D transmission, or from the BS. However, our work has three main different aspects from the previous work in [26]. Firstly, we consider a general conventional model, where any two close 
users are allowed to establish a D2D link, provided that the requested file of one user is cached in the memory of the other user. This is different from the cluster-based model in [26], where a cell is divided into multiple non-overlapping clusters and only the users in the same cluster are allowed to establish a D2D link. Thus, our proposed algorithm has a relaxed constraint on the location of the users in the cell and can potentially create more D2D communications among users. Secondly, we manage the mutual interference among different D2D links by efficiently scheduling the D2D links and fairly allocating the transmit power. However, [26] allows at most one D2D link to work in each cluster to avoid strong interference. Thirdly, we consider the max-min fairness of the scheduled D2D links to achieve perfect fairness and study the optimal power allocation at D2D transmitters. This is different from [26], where the fairness is ignored. As a result, the three differences lead to a completely different problem formulation and solution compared with that in [26]. To further clarity, our contributions are listed as follows.

- We consider the conventional model, where any two close users are allowed to establish a D2D link provided that the requested file of one user is cached in the memory of the other user. This creates more D2D communications among users compared with the cluster-based model in [26].

- We manage the mutual interference among different D2D links by efficiently scheduling the D2D links and fairly allocating the power allocation instead of dividing a cell into non-overlapping clusters and allowing at most one D2D link in each cluster in [26].

- We formulate a joint D2D link scheduling and power allocation problem to maximize the system throughput. Due to the non-convexity of the joint optimization problem, we decompose it into a D2D link scheduling problem and an optimal power allocation problem. Specifically, we intend to first maximize the number of the scheduled D2D links by solving the D2D link scheduling problem and then maximize the minimum transmission rate of the scheduled D2D links by solving the optimal power allocation problem.

- Numerical results indicate that both the number of the scheduled D2D links and the system throughput can be improved simultaneously with the Zipf-distribution caching scheme, the proposed D2D link scheduling algorithm, and the proposed optimal power allocation algorithm compared with the caching schemes and D2D link scheduling algorithms in [25] and [26]. 


\section{System MODEL}

We consider a cellular network with one BS and $K$ users, where the BS is located at the center of a cell and the users are randomly distributed in the cell as shown in Fig. 1, The BS has a file sever and stores $N$ files with equal size*, whose popularity probability follows Zipf distribution [29], i.e.,

$$
f_{\eta}=\frac{\frac{1}{\eta^{\gamma r}}}{\sum_{\zeta=1}^{N} \frac{1}{\zeta^{\gamma \gamma}}}, 1 \leq \eta \leq N,
$$

where $\eta$ is the file index, and $\gamma_{r}$ is the file request coefficient and controls the popularity distribution of files. Namely, a large $\gamma_{r}$ means that the first few files dominate the requests from users.

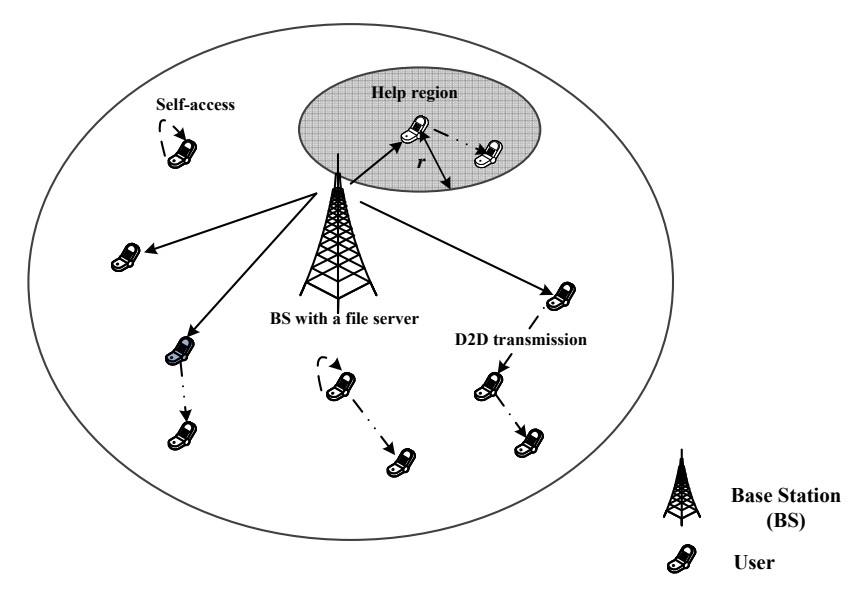

Figure 1. System model, where a user can obtain the requested file from its own memory (dash arrow), or from a helper through an one-hop D2D transmission (dash-dot arrow), or from the BS (solid arrow).

The file distribution by wireless caching consists of a placement phase and multiple delivery phases. In the placement phase, each user randomly and independently caches one out of $N$ 
files in its memory, according to the Zipf-distribution with a file caching coefficient $\gamma_{c} \dagger$. In each delivery phase, each user requests one file. If the requested file of a user can be found in its own memory, the user accesses the file without any transmission. On the other hand, if the user cannot find the requested file in its own memory, it can request the file either from a helper through an one-hop D2D transmission or from the BS. Here, any user in the cell can be a helper of a requesting user if two conditions are satisfied. One is that the two users are close in the cell. This is, the requesting user is in the help region, which is defined by a help distance $r$ around the helper. This may guarantee small pathloss and small transmit power of a D2D link on average. The other one is that the requested file is cached in the helper's memory. It should be noted that, a user may be served by more than one helpers. Then, the helper that can provide the highest transmission rate or SINR will be chosen as a transmitter in a D2D link. For clarity, the self-access, the one-hop D2D transmission, and the BS transmission are illustrated as dash arrow, dash-dot arrow, and solid arrow in Fig. 1, respectively, and the direction of a arrow denotes the transmission direction of a file.

We assume that all the D2D links share one WIFI channel and each D2D user works in half-duplex mode and receives or transmits wireless data in one time slot. We also assume that each user transmits data in the unicast mode and then cannot transmit data for multiple users simultaneously. This can be justified by two facts. One is that the asynchronous nature of the requested files, e.g., video clips. This means that the probability that different users request the same video clip from a common helper at the same time is negligible [14]. The other one is that the mobile nature of users. This makes it very hard to adopt the coded caching schemes which exploit the multicast opportunities between one transmitter and multiple receivers in [8]

\footnotetext{
${ }^{\dagger}$ We consider one file to simplify the presentation, although it is straightforward to generalize to multiple files with a Zipf distribution by caching each file independently. Note that the Zipf-distribution caching is not optimal for caching one file or multiple files. We use the Zipf distribution to cache files for four reasons. The first one is that, we focus on the algorithm design in the delivery phase and thus the proposed algorithms in this paper can be used for any caching scheme. The second one is that, the optimal caching distribution of the general model is quite hard to obtain (if it is possible). This is because the optimal caching scheme of one user is related to the number of its neighbors, which is defined as the users in its help region. In [24] and [25], a cell is divided into multiple non-overlapping clusters and the users in each cluster are assumed to be the neighbors of each other. Then, the numbers of neighbors for all the users in the same cluster are identical. This simplifies the mathematical derivation and makes it possible to obtain the optimal caching distribution for the cluster model. In our general model, the numbers of neighbors in terms of different users are quite different, especially for the users in the center of a cell and the users at the edge of a cell. This makes it quite hard to analyze the optimal caching distribution mathematically for a general model. Thus, a further study on the optimal caching scheme is beyond the scope of this paper. The third one is that, the Zipf distribution can well model the popularity of files [29]. Then, we use the Zipf distribution for wireless caching as a heuristic choice. The forth one is that, from the numerical results, the system performance (both the number of the scheduled D2D links and the system throughput) with the Zipf-distribution caching scheme and the proposed algorithms in this paper outperforms the system performance with the optimal caching distribution for the cluster model and the algorithms in [24] and [25].
} 
and [9]. In fact, the request of a video clip in most practical systems is implemented by a point to point (a transmitter to a receiver) transmission with a dedicated connection [24].

In the following, we will evaluate the SINR at D2D receivers. Suppose that $k_{\mathrm{S}}$ users in set $\mathcal{S}_{\mathrm{S}}$ can access their requested files from their own memory, and $k_{\mathrm{DB}}$ users in set $\mathcal{S}_{\mathrm{DB}}$ can obtain the requested files either from helpers through one-hop D2D transmissions or from the BS, and $k_{\mathrm{B}}$ users in set $\mathcal{S}_{\mathrm{B}}$ can only obtain the requested files from the BS. Define the link through which a user in $\mathcal{S}_{\mathrm{DB}}$ can obtain the requested file by D2D transmission as a potential D2D link and denote $l_{m}$ as the potential D2D link with receiver $m$. Without loss of generality, if we assume $\mathcal{S}_{\mathrm{DB}}=\left\{1,2, \cdots, k_{\mathrm{DB}}\right\}$, the potential D2D link set can be denoted as $\mathcal{L}_{\mathrm{DB}}=\left\{l_{1}, l_{2}, \cdots, l_{k_{\mathrm{DB}}}\right\}$.

Suppose that all the users in $\mathcal{S}_{\mathrm{DB}}$ are scheduled to work simultaneously and that the channel gain between the transmitter of the D2D link $l_{m}\left(m \in \mathcal{S}_{\mathrm{DB}}\right)$ to the receiver of the D2D link $l_{n}$ $\left(n \in \mathcal{S}_{\mathrm{DB}}\right)$ is $g(m, n)$. Then, the SINR at the user $m$ (D2D receiver) is

$$
v_{m}=\frac{p_{m} g(m, m)}{\sum_{n \in \mathcal{S}_{\mathrm{DB}}, n \neq m} p_{n} g(n, m)+N_{m}},
$$

where $p_{m}$ is the transmit power of the $\mathrm{D} 2 \mathrm{D}$ link $l_{m}$ and it is subject to the transmit power constraint $0 \leq p_{m} \leq p_{m}^{\max }$, and $N_{m}$ is the power of the additive white Gaussian noise (AWGN) at the receiver $m$. Here, we consider quality of experience (QoE) guaranteed D2D transmissions. Thus, the SINR at each D2D receiver is required to be greater than or equal to the minimum acceptable SINR, i.e., $v_{m} \geq \breve{v}_{m}$, which is related to the modulated and encoding scheme at each D2D transmitter.

\section{PRoblem Formulation AND ANALYSiS}

\section{A. Problem Formulation}

From the previous section, there is a chance that not all the potential D2D links in $\mathcal{L}_{\mathrm{DB}}$ can be scheduled simultaneously for two reasons. One reason is that the potential D2D links in $\mathcal{L}_{\mathrm{DB}}$ may share the same users. That is, one user may be a D2D transmitter in one potential D2D link and a D2D receiver in another potential D2D link. Then, the two links cannot work simultaneously. The other reason is that, some potential D2D links cannot be satisfied with the minimum acceptable SINRs. Thus, we intend to develop a joint D2D link scheduling and power 
allocation algorithm to maximize the system throughput. Formally, we have

$$
\begin{aligned}
\left(\mathrm{P}_{0}\right): & \max _{p_{m}: m \in \mathcal{S}_{\mathrm{D}}} \sum_{m \in \mathcal{S}_{\mathrm{D}}} \log \left(v_{m}+1\right) \\
\text { s.t. } & \mathcal{S}_{\mathrm{D}} \subset \mathcal{S}_{\mathrm{DB}}, \\
& \mathcal{S}_{\mathrm{DT}} \cap \mathcal{S}_{\mathrm{D}}=\varnothing, \\
& v_{m} \geq \check{v}_{m}, \forall m \in \mathcal{S}_{\mathrm{D}}, \\
& 0 \leq p_{m} \leq p_{m}^{\max }, \forall m \in \mathcal{S}_{\mathrm{D}},
\end{aligned}
$$

where the scheduled D2D link set $\mathcal{S}_{\mathrm{D}}$ and the corresponding transmit power $p_{m}, \forall m \in \mathcal{S}_{\mathrm{D}}$, are the variables to be optimized, $\mathcal{S}_{\mathrm{DT}}$ is the $\mathrm{D} 2 \mathrm{D}$ transmitter set corresponding to the $\mathrm{D} 2 \mathrm{D}$ receivers in $\mathcal{S}_{\mathrm{D}}$, and the constraint (4) guarantees that different scheduled D2D links do not share the same D2D users.

Unfortunately, the objective function of $\left(\mathrm{P}_{0}\right)$ is non-convex. To obtain the joint optimal D2D link scheduling and power allocation solution, the complexity of the exhaustive search is quite high $屯$. Alternatively, we seek to obtain a suboptimal solution of $\left(\mathrm{P}_{0}\right)$ with reasonable complexity. Briefly, we intend to schedule the D2D links with strong communication channels and weak interference channels and allocate the power to the scheduled D2D links fairly. Thus, we decompose $\left(\mathrm{P}_{0}\right)$ into two subproblems $\$$, i.e., a D2D link scheduling problem and an optimal power allocation problem. For the first subproblem, we intend to optimize $\mathcal{S}_{\mathrm{D}}$, to maximize the number of D2D links satisfying both the SINR and the transmit power constraints. For the second subproblem, we intend to optimize $p_{m}, \forall m \in \mathcal{S}_{\mathrm{D}}$, to maximize the minimum transmission rate of the scheduled D2D links. In what follows, we will formulate the two subproblems.

1) D2D Link Scheduling Problem: To maximize the number of the scheduled D2D links satisfying both the SINR and the transmit power constraints, the D2D link scheduling problem

\footnotetext{
${ }^{\ddagger}$ The complexity of the exhaustive search is $O\left(\Pi_{m=1}^{m=k_{\mathrm{DB}}} \frac{p_{m}^{\max }}{\epsilon}\right)$, where $p_{m}^{\max }$ is the maximum transmit power of the D2D link $l_{m}, \epsilon$ is the maximum tolerance error of the exhaustive search scheme, and $k_{\mathrm{DB}}$ is the number of users that can obtain the requested files either from helpers through one-hop D2D transmissions or from the BS. Suppose that $k_{\mathrm{DB}}=20, p_{m}^{\max }=p_{n}^{\max }=$ $p^{\max }=100 \mathrm{mw}, \forall m \neq n$, and $\epsilon=0.01$. Then, the complexity of the exhaustive search is extremely large, i.e., $O\left(10000^{20}\right)$.

${ }^{\S}$ Note that the decomposition is not optimal and finding the optimal decomposition will be our future work (if it is feasible).
} 
can be written as

$$
\begin{aligned}
\left(\mathrm{P}_{1}\right): & \max _{\mathcal{S}_{\mathrm{D}} \subset \mathcal{S}_{\mathrm{DB}}}\left|\mathcal{S}_{\mathrm{D}}\right| \\
\text { s.t. } & (4),(6), \\
& v_{m} \geq \bar{v}_{m}, \forall m \in \mathcal{S}_{\mathrm{D}},
\end{aligned}
$$

where $\left|\mathcal{S}_{\mathrm{D}}\right|$ is the cardinality of $\mathcal{S}_{\mathrm{D}}$ and $\bar{v}_{m}=\check{v}_{m}$. It is clear that the solution of $\left(\mathrm{P}_{1}\right)$ may maximize the number of the scheduled D2D links. However, scheduling the largest number of D2D links may lead to small system throughput since more D2D links may cause more mutual interference. To deal with this issue, we introduce a scheduling coefficient $c_{\mathrm{s}}(\mathrm{dB})$ and replace $\bar{v}_{m}=\breve{v}_{m}$ with $\bar{v}_{m}=\max \left\{\check{v}_{m}, c_{\mathrm{s}}\right\}$. Then, if $c_{s} \leq \min _{m \in \mathcal{S}_{\mathrm{DB}}} \check{v}_{m}$, we have $\bar{v}_{m}=\check{v}_{m}$. Then, $\left(\mathrm{P}_{1}\right)$ maximizes the number of the scheduled D2D links. As we increase $c_{\mathrm{s}}$, the SINR constraint (7) becomes strict, only the D2D links with strong communication channels and weak interference channels are scheduled. This potentially increases the system throughput. Thus, we can numerically choose the value of $c_{s}$ to obtain an acceptable number of the scheduled D2D links as well as the system throughput.

Theorem 1: Each transmit power $p_{n}, \forall n \in \mathcal{S}_{\mathrm{D}}$, increases if any $\operatorname{SINR} v_{m}, m \in \mathcal{S}_{\mathrm{D}}$, in $\left(\mathrm{P}_{1}\right)$ increases. Meanwhile, the value of $\left|\mathcal{S}_{\mathrm{D}}\right|$ remains constant or decreases if any $\operatorname{SINR} v_{m}, m \in \mathcal{S}_{\mathrm{D}}$, in $\left(\mathrm{P}_{1}\right)$ increases.

Proof: The proof is provided in Appendix A.

From Theorem 1, the value of $\left|\mathcal{S}_{\mathrm{D}}\right|$ may decrease if any SINR $v_{m}$ in $\mathcal{S}_{\mathrm{D}}$ increases. Then, problem $\left(\mathrm{P}_{1}\right)$ is equivalent to

$$
\begin{aligned}
& \left(\mathrm{P}_{2}\right): \max _{\mathcal{S}_{\mathrm{D}} \subset \mathcal{S}_{\mathrm{DB}}}\left|\mathcal{S}_{\mathrm{D}}\right| \\
& \text { s.t. } \quad(4),(6), \\
& \quad v_{m}=\bar{v}_{m}, \forall m \in \mathcal{S}_{\mathrm{D}} .
\end{aligned}
$$

By solving problem $\left(\mathrm{P}_{2}\right)$, we may obtain the optimal D2D receiver set $\mathcal{S}_{\mathrm{D}}^{*}$ and the corresponding D2D link set $\mathcal{L}_{\mathrm{D}}^{*}$. Without loss of generality, we assume $\mathcal{S}_{\mathrm{D}}^{*}=\left\{1,2, \cdots, k_{\mathrm{D}}\right\}$ and $\mathcal{L}_{\mathrm{D}}^{*}=$ $\left\{l_{1}, l_{2}, \cdots, l_{k_{\mathrm{D}}}\right\}$. It is clear that problem $\left(\mathrm{P}_{2}\right)$ outputs the SINR vector $\overline{\mathbf{V}}=\left[\bar{v}_{1}, \bar{v}_{2}, \cdots, \bar{v}_{k_{\mathrm{D}}}\right]^{\mathrm{T}}$ at the scheduled D2D receivers. Accordingly, we assume the power allocation vector is $\overline{\mathbf{P}}=$ $\left[\bar{p}_{1}, \bar{p}_{2}, \cdots, \bar{p}_{k_{\mathrm{D}}}\right]^{\mathrm{T}}$. 
2) Optimal Power Allocation Problem: From Theorem 1 again, the value of $\left|\mathcal{S}_{\mathrm{D}}^{*}\right|$ may remain constant if any SINR $v_{m}, m \in \mathcal{S}_{\mathrm{D}}^{*}$, increases. Thus, we may improve the minimum transmission rate or the minimum SINR of the scheduled D2D links without compromising $\left|\mathcal{S}_{\mathrm{D}}^{*}\right|$, provided that the transmit power constraints of the scheduled D2D links are not violated, i.e.,

$$
\begin{array}{ll}
\left(\mathrm{P}_{3}\right): & \max _{p_{m}: m \in \mathcal{S}_{\mathrm{D}}^{*} 1 \leq m \leq k_{\mathrm{D}}} \min \left(v_{m}+1\right) \\
\text { s.t. } & v_{m} \geq \bar{v}_{m}, \forall m \in \mathcal{S}_{\mathrm{D}}^{*}, \\
& \bar{p}_{m} \leq p_{m} \leq p_{m}^{\max }, \forall m \in \mathcal{S}_{\mathrm{D}}^{*} .
\end{array}
$$

\section{B. Analysis of Subproblem $\left(P_{2}\right)$}

To solve problem $\left(\mathrm{P}_{2}\right)$, we shall develop a D2D link scheduling algorithm to select the largest number of D2D links satisfying all the constraints in problem $\left(\mathrm{P}_{2}\right)$. In fact, problem $\left(\mathrm{P}_{2}\right)$ is an admission control problem (i.e., link scheduling problem) [32]-[35]. However, it is different from a regular admission control problem. In the regular admission control problem, one transmitter has one dedicated receiver. In our system model, there is a chance that one user is the transmitter in one potential D2D link and the receiver of another potential D2D link. Since the scheduled D2D links cannot share the same D2D users, i.e., the constraint (4), problem $\left(\mathrm{P}_{2}\right)$ is more complicate than the regular admission control problem and the algorithm for the regular admission control problem cannot be directly used to solve problem $\left(\mathrm{P}_{2}\right)$. Thus, we need to develop a scheduling algorithm to solve problem $\left(\mathrm{P}_{2}\right)$.

Denote the events that any two potential D2D links in a potential D2D link set do not share the same users and that all the minimum SINR constraints in a potential D2D link set can be satisfied as $C_{1}$ and $C_{2}$. Accordingly, $\bar{C}_{1}$ and $\bar{C}_{2}$ denote the events that $C_{1}$ and $C_{2}$ cannot be satisfied, respectively. Then, we have the following three cases depending on whether $C_{1}$ and/or $C_{2}$ can be satisfied in the potential D2D link set $\mathcal{L}_{\mathrm{DB}}$.

Case I: $\left(C_{1}, C_{2}\right)$. In this case, all the potential D2D links in $\mathcal{L}_{\mathrm{DB}}$ will be scheduled. Then, we have $\mathcal{S}_{\mathrm{D}}^{*}=\mathcal{S}_{\mathrm{DB}}$.

Case II: $\left(C_{1}, \bar{C}_{2}\right)$. In this case, problem $\left(\mathrm{P}_{2}\right)$ reduces to a regular admission control problem. To solve this problem, the fewest D2D links in $\mathcal{L}_{\mathrm{DB}}$ shall be removed until $C_{2}$ can be satisfied.

Case III: $\left(\bar{C}_{1}\right)$. In this case, some D2D links share the same users and are not allowed to work simultaneously. To solve this problem, we shall first divide the potential D2D links in $\mathcal{L}_{\mathrm{DB}}$ into 
different groups (or subsets), such that any two potential D2D links in one group do not share the same users. After that, each group becomes case I or case II.

For generality, we will consider Case III for problem $\left(\mathrm{P}_{2}\right)$ in the rest of this paper. By analyzing problem $\left(\mathrm{P}_{2}\right)$, we have

Theorem 2: Problem $\left(\mathrm{P}_{2}\right)$ is NP-hard.

Proof: The proof is provided in Appendix B.

Considering the highly computational complexity of obtaining the optimal solution for a NPhard problem, we will develop an efficient algorithm to obtain a suboptimal solution of problem $\left(\mathrm{P}_{2}\right)$ in what follows.

\section{Analysis of Subproblem $\left(P_{3}\right)$}

Once the optimal D2D set $\mathcal{S}_{\mathrm{D}}^{*}$ is obtained from problem $\left(\mathrm{P}_{2}\right)$, problem $\left(\mathrm{P}_{3}\right)$ is a feasible max-min optimization problem with transmit power of D2D links as variables. Then, we will

first analyze the property of the optimal power allocation of problem $\left(\mathrm{P}_{3}\right)$ and then develop an optimal power allocation algorithm to achieve the optimal performance.

\section{D2D LINK SCHEDULING}

In this section, we aim to maximize the number of the scheduled D2D links. However, some of the potential D2D links may be dependent. That is, different potential D2D links in $\mathcal{L}_{\mathrm{DB}}$ share the same users. This is the main difference between problem $\left(\mathrm{P}_{2}\right)$ and a regular admission control problem, where any two different potential D2D links do not share the same users. Thus, we shall divide the potential D2D links in $\mathcal{L}_{\mathrm{DB}}$ into different groups, such that different potential D2D links in each group are independent. In this way, problem $\left(\mathrm{P}_{2}\right)$ is decomposed into several subproblems corresponding to different groups. After maximizing the number of the potential D2D links that satisfying both $C_{1}$ and $C_{2}$ for each subproblem (group), we can obtain a suboptimal solution of problem $\left(\mathrm{P}_{2}\right)$.

In what follows, we will first divide the potential D2D links in $\mathcal{L}_{\mathrm{DB}}$ into different groups such that the potential D2D links in each group are independent and satisfy $C_{1}$. Then, we will maximize the number of the potential D2D links satisfying $C_{2}$ in each group. Specifically, we develop a centralized power control (CPC) algorithm to check the state of each group, i.e., to decide whether the potential D2D links in each group satisfy $C_{2}$ or not. If the potential 
D2D links in one group do not satisfy $C_{2}$, we further develop a removal algorithm to remove the fewest potential D2D links from this group. After applying the CPC algorithm and/or the removal algorithm into each group, the largest number of the potential D2D links satisfying both $C_{1}$ and $C_{2}$ in each group is obtained. Finally, the potential D2D links in the group with the largest number of potential D2D links will be scheduled and other potential D2D links will not be allowed.

\section{A. Potential D2D Links Division}

To maximize the number of the scheduled D2D links, we shall add as many potential D2D links as possible into each group. This leads to the fewest groups. Thus, the division of the potential D2D links can be transferred to an edge coloring problem in graph theory, where the edges in a graph are colored with the fewest colors while guaranteeing that any two edges sharing the same vertex are colored differently.

Denote the connection among the users involved the potential D2D links in $\mathcal{L}_{\mathrm{DB}}$ as a graph $G(\mathcal{O}, \mathcal{E})$, where $\mathcal{O}$ is the vertex set denoting the involved users in $\mathcal{L}_{\mathrm{DB}}, \mathcal{E}$ is the edge set denoting the D2D links in $\mathcal{L}_{\mathrm{DB}}$. Then, we may color the edges in $\mathcal{E}$ with $\chi^{\prime}(G)$ colors, which is the edge chromatic number [30]. Since the edge coloring problem is NP-hard for a general graph, it is non-trivial to obtain the optimal solution. Alternatively, we develop an iterative edge-coloring algorithm as shown in Algorithm 1, which colors the edges in $\mathcal{E}$ with $N_{\mathrm{C}}$ colors $\left(N_{\mathrm{C}} \geq \chi^{\prime}(G)\right)$. More formally, denote $\mathcal{E}=\mathcal{E}_{1} \cup \ldots \cup \mathcal{E}_{N_{\mathrm{C}}}$, where $\mathcal{E}_{i}\left(1 \leq i \leq N_{\mathrm{C}}\right)$ represents the edge set with the $i$ th color after edge coloring. We assume that there are $k_{i}$ edges in $\mathcal{E}_{i}$, which corresponds to $k_{i}$ potential D2D links. If we denote the receiver set of the $k_{i}$ potential D2D links as $\mathcal{S}_{i}=\left\{i_{1}, i_{2}, \cdots, i_{k_{i}}\right\}$, the $k_{i}$ potential D2D link set can be denoted as $\mathcal{L}_{i}=\left\{l_{i_{1}}, l_{i_{2}}, \cdots, l_{i_{k_{i}}}\right\}$.

In the $i$ th iteration of Algorithm 1 the maximum matching $\Phi$ is obtained by solving a linear

\footnotetext{
๑The maximum matching is the maximum edge set, where any two edges do not share a common vertex.
} 
programming problem [31]

$$
\begin{aligned}
\left(\mathrm{P}_{4}\right): & \max _{x_{e}: e \in \mathcal{E}} \sum_{e \in \mathcal{E}} x_{e} \\
\text { s.t. } & \sum_{e \sim o} x_{e} \leq 1, \forall o \in \mathcal{O}, \\
& 0 \leq x_{e} \leq 1, \forall e \in \mathcal{E},
\end{aligned}
$$

where $e \sim o$ means that the edge $e$ is incident on the vertex $o$, and rounding the solutions $x_{e}$ $(e \in \mathcal{E})$ to 0 or 1 in order to map the solutions to integers. That is, $x_{e}=0$ if $0 \leq x_{e}<0.5$ and $x_{e}=1$ if $0.5 \leq x_{e} \leq 1$. In this way, the edges $x_{e}=1$ are colored with the $i$ th color and put into the set $\mathcal{L}_{i}=\left\{l(e): x_{e}=1, e \in \mathcal{E}\right\}$, where $l(e)$ denotes the D2D link corresponding to the edge $e$. This procedure is terminated until all the edges are colored. Note that the computational complexity of Algorithm 1 is dominated by solving the linear programming problem $\left(\mathrm{P}_{4}\right)$ with computational complexity $O\left(k_{\mathrm{DB}}^{3}\right)$ [36]. Thus, the overall computational complexity of Algorithm 1 is $O\left(k_{\mathrm{DB}}^{3}\right)$.

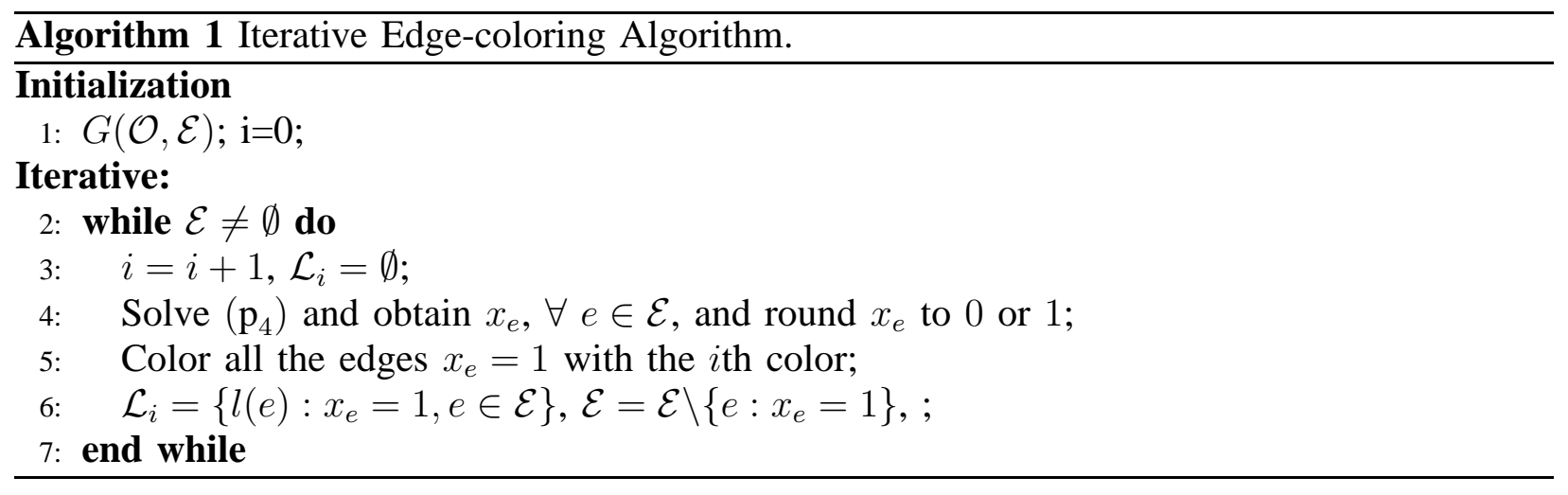

\section{B. CPC Algorithm}

After dividing the potential D2D links into $\mathcal{L}_{i}\left(1 \leq i \leq N_{\mathrm{C}}\right)$ with the iterative edge-coloring algorithm, $C_{1}$ is satisfied among the potential D2D links in each $\mathcal{L}_{i}$. In what follows, we will develop an algorithm to check the state of the potential D2D links in $\mathcal{L}_{i}$, i.e., to decide whether the potential D2D links in $\mathcal{L}_{i}$ satisfy $C_{2}$ or not.

If all the potential D2D links in $\mathcal{L}_{i}$ satisfy $C_{2}$, there exists a power allocation to satisfy all the potential D2D links in $\mathcal{L}_{i}$ with the minimum SINR constraints $\overline{\mathbf{V}}_{i}=\left[\begin{array}{lll}\bar{v}_{i_{1}}, & \bar{v}_{i_{2}}, \ldots, \bar{v}_{i_{k_{i}}}\end{array}\right]^{\mathrm{T}}$. 
Suppose that the power allocation is $\overline{\mathbf{P}}_{i}=\left[\bar{p}_{i_{1}}, \bar{p}_{i_{2}}, \ldots, \bar{p}_{i_{k_{i}}}\right]^{\mathrm{T}}$. Then, we have

$$
H_{i}\left(\overline{\mathbf{V}}_{i}\right) \overline{\mathbf{P}}_{i}=\mathbf{N}_{i}
$$

where

$$
H_{i}\left(\overline{\mathbf{V}}_{i}\right)=\left[\begin{array}{cccc}
g\left(i_{1}, i_{1}\right) / \bar{v}_{i_{1}}-g\left(i_{1}, i_{2}\right) & \cdots & -g\left(i_{1}, i_{k_{i}}\right) \\
-g\left(i_{2}, i_{1}\right) & g\left(i_{2}, i_{2}\right) / \bar{v}_{i_{2}} & \cdots & -g\left(i_{2}, i_{k_{i}}\right) \\
\vdots & \vdots & \ddots & \vdots \\
-g\left(i_{k_{i}}, i_{1}\right) & -g\left(i_{k_{i}}, i_{2}\right) & \cdots & g\left(i_{k_{i}}, i_{k_{i}}\right) / \bar{v}_{i_{k_{i}}}
\end{array}\right]^{\mathrm{T}}
$$

and

$$
\mathbf{N}_{i}=\left[N_{i_{1}}, N_{i_{2}}, \cdots, N_{i_{k_{i}}}\right]^{\mathrm{T}}
$$

Then, the power allocation $\overline{\mathbf{P}}_{i}$ can be derived as

$$
\overline{\mathbf{P}}_{i}=H_{i}^{-1}\left(\overline{\mathbf{V}}_{i}\right) \mathbf{N}_{i}
$$

If the potential D2D links in $\mathcal{L}_{i}$ satisfy $C_{2}$, we have $\mathbf{0} \preceq \overline{\mathbf{P}}_{i} \preceq \mathbf{P}_{i}^{\max }$, where $\mathbf{P}_{i}^{\max }=$ $\left[p_{i_{1}}^{\max }, p_{i_{2}}^{\max }, \cdots, p_{i_{k_{i}}}^{\max }\right]^{\mathrm{T}}, \mathbf{0}$ is a $k_{i} \times 1$ vector with all zero elements, and " $\preceq$ " is an elementwise operator.

Consequently, we have the CPC algorithm as

- the potential D2D links in $\mathcal{L}_{i}$ satisfy $C_{2}$ if $\mathbf{0} \preceq \overline{\mathbf{P}}_{i} \preceq \mathbf{P}_{i}^{\max }$ holds.

- the potential D2D links in $\mathcal{L}_{i}$ do not satisfy $C_{2}$ if $\mathbf{0} \preceq \overline{\mathbf{P}}_{i} \preceq \mathbf{P}_{i}^{\max }$ does not holds.

In our algorithm, the BS first collects the channel gains among different potential D2D links and the minimum SINR constraint of each potential D2D link, and then calculates the required power allocation to satisfy the potential D2D links in $\mathcal{L}_{i}$ with their minimum SINR constraints. Thus, we name this algorithm as centralized power control (CPC) algorithm. Essentially, the main ideas of the CPC algorithm and the distributed constrain power control (DCPC) algorithm in [32] are similar: check whether multiple transceivers can be supported simultaneously by checking whether the transmit power constraints are violated to achieve the minimum SINR constraints. However, the CPC algorithm is more efficient than the DCPC algorithm to implement. More specifically, to implement the DCPC algorithm, each D2D transmitter in $\mathcal{L}_{i}$ iteratively adapts its transmit power to achieve the minimum SINR constraint until all the transmit power converges. If all the minimum SINR constraints are satisfied after transmit power converges, the potential 
D2D links in $\mathcal{L}_{i}$ satisfy $C_{2}$. If some minimum SINR constraints are not satisfied, the potential D2D links in $\mathcal{L}_{i}$ do not satisfy $C_{2}$. Then, a removal algorithm will be developed to remove some potential D2D links from $\mathcal{L}_{i}$. In the CPC algorithm, the BS first collects all the necessary information and then calculates the power allocation to achieve the minimum SINR constraints. If the calculated power allocation can be satisfied at all the D2D transmitters in $\mathcal{L}_{i}$, the potential D2D links in $\mathcal{L}_{i}$ satisfy $C_{2}$. Otherwise, a removal algorithm will be developed.

\section{Removal Algorithm}

From the CPC algorithm, there exists at least one calculated transmit power $\bar{p}_{i_{m}}$ in $\mathcal{L}_{i}$ satisfying $\bar{p}_{i_{m}}>p_{i_{m}}^{\max }$ or $\bar{p}_{i_{m}}<0$ if the potential D2D links in $\mathcal{L}_{i}$ do not satisfy $C_{2}$. This is because the mutual interference among the potential D2D links in $\mathcal{L}_{i}$ is too strong. Then, we shall remove some potential D2D links from $\mathcal{L}_{i}$ to enable the remaining ones to satisfy $C_{2}$. Our approach is to remove the potential D2D link which is likely to cause the strongest interference to others or receive the strongest interference from others each time until the remaining potential D2D links satisfy $C_{2}$.

Specifically, to satisfy the minimum SINR constraint $\bar{v}_{i_{m}}\left(i_{m} \in \mathcal{S}_{i}\right)$, the transmitter of the potential D2D link $l_{i_{m}}$ has to set the transmit power no less than $\frac{N_{i_{m}} \bar{v}_{i_{m}}}{g\left(i_{m}, i_{m}\right)}$ and causes no less than $\frac{N_{i_{m}} \bar{v}_{i_{m}}}{g\left(i_{m}, i_{m}\right)} g\left(i_{m}, i_{n}\right)\left(i_{n} \in \mathcal{S}_{i}, i_{n} \neq i_{m}\right)$ interference to another potential D2D link $l_{i_{n}}$. Since a potential D2D link with a smaller minimum SINR constraint and a larger maximum transmit power can tolerate more interference from other potential D2D links, we define the relative interference from $l_{i_{m}}$ to $l_{i_{n}}$ as $I_{r}\left(l_{i_{m}}, l_{i_{n}}\right)=\frac{\bar{v}_{i_{n}}}{p_{i_{n}} \frac{N_{i_{m}}}{g\left(i_{m}, i_{m}\right)}} g\left(i_{m}, i_{n}\right)$. Then, the summation of relative interference generated by $l_{i_{m}}$ is larger than

$$
\alpha_{i_{m}}=\sum_{n=1, n \neq m}^{n=k_{i}} I_{r}\left(l_{i_{m}}, l_{i_{n}}\right)=\frac{N_{i_{m}} \bar{v}_{i_{m}}}{g\left(i_{m}, i_{m}\right)} \sum_{n=1, n \neq m}^{n=k_{i}} \frac{\bar{v}_{i_{n}}}{p_{i_{n}}^{\max }} g\left(i_{m}, i_{n}\right) .
$$

Similarly, to satisfy the minimum SINR constraint $\bar{v}_{i_{n}}\left(i_{n} \in \mathcal{S}_{i}, i_{n} \neq i_{m}\right)$, the transmitter of the potential D2D link $l_{i_{n}}$ has to set the transmit power no less than $\frac{N_{i_{n}} \bar{i}_{n}}{g\left(i_{n}, i_{n}\right)}$. Then, the summation of relative interference to the potential D2D link $l_{i_{m}}$ is larger than

$$
\beta_{i_{m}}=\sum_{n=1, n \neq m}^{n=k_{i}} I_{r}\left(l_{i_{n}}, l_{i_{m}}\right)=\frac{\bar{v}_{i_{m}}}{p_{i_{m}}^{\max }} \sum_{n=1, n \neq m}^{n=k_{i}} \frac{N_{i_{n}} \bar{v}_{i_{n}}}{g\left(i_{n}, i_{n}\right)} g\left(i_{n}, i_{m}\right) .
$$


Thus, the potential D2D link $l_{i_{m^{*}}}$, where

$$
i_{m^{*}}=\underset{1 \leq m \leq k_{i}}{\arg \max } \max \left\{\alpha_{i_{m}}, \beta_{i_{m}}\right\}
$$

is likely to cause the strongest interference to others or receive the strongest interference from others and will be removed from $\mathcal{L}_{i}$.

It should be noted that, the proposed removal algorithm is different from the DCPC-based removal algorithm in [32]. Specifically, our proposed algorithm utilizes the information of the maximum transmit power at each transmitter while the DCPC-based removal algorithm in [32] utilizes the converged transmit power of the DCPC algorithm, although the two algorithms share other two kinds of information, i.e., the minimum SINR constraints and the interference channel gains among different potential D2D links. Besides, our proposed removal algorithm depends on the relative interference among different transmissions whereas the DCPC-based removal algorithm in [32] is developed by measuring the absolute interference after the DCPC algorithm converges. Thus, the calculated interference in our proposed algorithm is more accurate than that in [32] and our proposed removal algorithm is more flexible than the DCPC-based removal algorithm in [32]. In fact, from the numerical results in Section VI, our proposed removal algorithm outperforms the DCPC-based removal algorithm.

\section{Scheduling Algorithm and Complexity Analysis}

After applying the CPC algorithm and/or removal algorithm into each potential D2D link set $\mathcal{L}_{i}\left(1 \leq i \leq N_{\mathrm{C}}\right)$, the potential D2D links in each $\mathcal{L}_{i}$ satisfy both $C_{1}$ and $C_{2}$. Then, the potential D2D links in $\mathcal{L}_{i^{*}}=\underset{1 \leq i \leq N_{C}}{\arg \max }\left|\mathcal{L}_{i}\right|$ with the largest number of the potential D2D links will be scheduled. To summarize, we illustrate the detail D2D link scheduling algorithm in Algorithm 2 .

Note that Algorithm 2 consists of Algorithm 1 the CPC algorithm, and the removal algorithm. The computational complexity of Algorithm 1 is $O\left(k_{\mathrm{DB}}^{3}\right)$. The computational complexity of the CPC algorithm is dominated by calculating (16), i.e., $\overline{\mathbf{P}}_{i}=H_{i}^{-1}\left(\overline{\mathbf{V}}_{i}\right) \mathbf{N}_{i}$. In the worst case, $H_{i}\left(\overline{\mathbf{V}}_{i}\right)$ is a $k_{\mathrm{DB}} \times k_{\mathrm{DB}}$ matrix and $\mathbf{N}_{i}$ is a $k_{\mathrm{DB}} \times 1$ vector. The computational complexity of the inverse of a $k_{\mathrm{DB}} \times k_{\mathrm{DB}}$ matrix $H_{i}\left(\overline{\mathbf{V}}_{i}\right)$ is $O\left(k_{\mathrm{DB}}^{2.373}\right)$ and the computational complexity of the multiplication of a $k_{\mathrm{DB}} \times k_{\mathrm{DB}}$ matrix $H_{i}^{-1}\left(\overline{\mathbf{V}}_{i}\right)$ and a $k_{\mathrm{DB}} \times 1$ vector $\mathbf{N}_{i}$ is $O\left(k_{\mathrm{DB}}^{2}\right)$ [37]. Then, the computational complexity of the $\mathrm{CPC}$ algorithm is $O\left(k_{\mathrm{DB}}^{2.373}\right)+O\left(k_{\mathrm{DB}}^{2}\right)=O\left(k_{\mathrm{DB}}^{2.373}\right)$. Besides, 
the computational complexity of the removal algorithm is dominated by arithmetic and thus is $O\left(k_{\mathrm{DB}}\right)$. Consequently, the computational complexity of Algorithm $\mathbf{2}$ is $O\left(k_{\mathrm{DB}}^{3}\right)+O\left(k_{\mathrm{DB}}^{2.373}\right)+$ $O\left(k_{\mathrm{DB}}\right)=O\left(k_{\mathrm{DB}}^{3}\right)$.

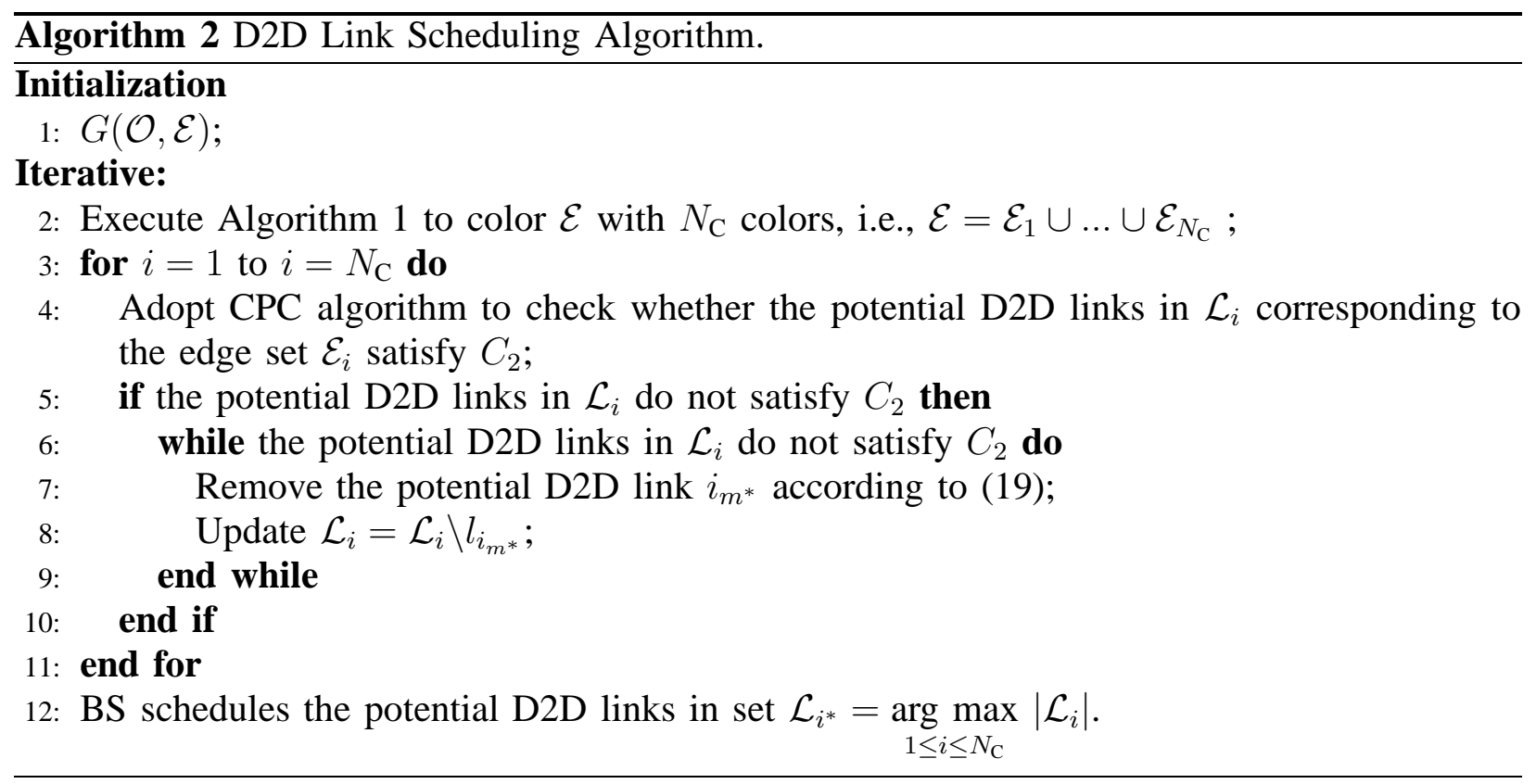

\section{Optimization of Power Allocation}

In the previous section, we have solved problem $\left(\mathrm{P}_{2}\right)$ and obtained the scheduled D2D link set $\mathcal{L}_{i^{*}}=\mathcal{L}_{\mathrm{D}}^{*}=\left\{l_{1}, l_{2}, \cdots, l_{k_{\mathrm{D}}}\right\}$ and the D2D receiver set $\mathcal{S}_{\mathrm{D}}^{*}=\left\{1,2, \cdots, k_{\mathrm{D}}\right\}$. Accordingly, we may obtain the output transmit power vector and the SINR vector as $\overline{\mathbf{P}}_{i^{*}}=\overline{\mathbf{P}}=\left[\bar{p}_{1}, \bar{p}_{2}, \cdots, \bar{p}_{k_{\mathrm{D}}}\right]^{\mathrm{T}}$ and $\mathbf{V}_{i^{*}}=\overline{\mathbf{V}}=\left[\bar{v}_{1}, \bar{v}_{2}, \cdots, \bar{v}_{k_{\mathrm{D}}}\right]^{\mathrm{T}}$ from problem $\left(\mathrm{P}_{2}\right)$. In this section, we will solve problem $\left(\mathrm{P}_{3}\right)$ to maximize the minimum transmission rate or the minimum SINR of the scheduled D2D links without compromising the number of the scheduled D2D links. Although problem $\left(\mathrm{P}_{3}\right)$ can be transformed to a convex problem and is solved with a convex optimization toolbox, we seek to solve it analytically to shed more light on the optimal power allocation. Specifically, we will first analyze the property of the optimal solution of problem $\left(\mathrm{P}_{3}\right)$. Then, we will develop a binary-search based power allocation to obtain the optimal power allocation. Finally, we analyze the computational complexity of our algorithm. 


$$
\begin{aligned}
& H\left(\mathbf{V}^{(m)}\left(\bar{v}_{m}\right)\right)= \\
& {\left[\begin{array}{ccccccc}
g(1,1) / \bar{v}_{m} & -g(1,2) & \cdots & -g(1, m) & -g(1, m+1) & \cdots & -g\left(1, k_{\mathrm{D}}\right) \\
-g(2,1) & g(2,2) / \bar{v}_{m} & \cdots & -g(2, m) & -g(2, m+1) & \cdots & -g\left(2, k_{\mathrm{D}}\right) \\
\vdots & \vdots & \ddots & \vdots & \vdots & \ddots & \vdots \\
-g(m, 1) & -g(m, 2) & \cdots & g(m, m) / \bar{v}_{m} & -g(m, m+1) & \cdots & -g\left(m, k_{\mathrm{D}}\right) \\
-g(m+1,1) & -g(m+1,2) & \cdots & -g(m+1, m) & g(m+1, m+1) / \bar{v}_{m+1} & \cdots & -g\left(m+1, k_{\mathrm{D}}\right) \\
\vdots & \vdots & \ddots & \vdots & \vdots & \ddots & \vdots \\
-g\left(k_{\mathrm{D}}, 1\right) & -g\left(k_{\mathrm{D}}, 2\right) & \cdots & -g\left(k_{\mathrm{D}}, m\right) & -g\left(k_{\mathrm{D}}, m+1\right) & \cdots & g\left(k_{\mathrm{D}}, k_{\mathrm{D}}\right) / \bar{v}_{k_{\mathrm{D}}}
\end{array}\right]^{\mathrm{T}}}
\end{aligned}
$$

\section{A. Binary-search based Power Allocation}

As mentioned above, the minimum SINR constraints $\mathbf{V}=\overline{\mathbf{V}}=\left[\bar{v}_{1}, \bar{v}_{2}, \cdots, \bar{v}_{k_{\mathrm{D}}}\right]^{\mathrm{T}}$ of the scheduled D2D links are satisfied with the power allocation $\overline{\mathbf{P}}=\left[\bar{p}_{1}, \bar{p}_{2}, \cdots, \bar{p}_{k_{\mathrm{D}}}\right]^{\mathrm{T}}$. To further increase the SINRs in $\mathbf{V}$ and maximize the minimum SINR in $\mathbf{V}$, i.e., problem $\left(\mathrm{P}_{3}\right)$, we shall increase the SINRs in $\mathrm{V}$ with small minimum SINR constraints with priority.

Intuitively, if we assume $\bar{v}_{1} \leq \bar{v}_{2} \leq \cdots \leq \bar{v}_{k_{\mathrm{D}}}$, we shall increase $v_{1}$ from $\bar{v}_{1}$ to $\bar{v}_{2}$, i.e., $\bar{v}_{1} \leq v_{1} \leq \bar{v}_{2}$, and then increase $v_{1}$ and $v_{2}$ from $\bar{v}_{2}$ to $\bar{v}_{3}$, i.e., $\bar{v}_{2} \leq v_{1}=v_{2} \leq \bar{v}_{3}$, and then increase $v_{1}, v_{2}, \cdots, v_{m}\left(1 \leq m<k_{\mathrm{D}}\right)$ from $\bar{v}_{m}$ to $\bar{v}_{m+1}$, i.e., $\bar{v}_{m} \leq v_{1}=v_{2}=\cdots=v_{m} \leq \bar{v}_{m+1}$. This procedure is terminated until some maximum transmit power constraints are violated. In this way, we maximize the minimum SINR of the scheduled D2D links. More formally, we have the following Theorem.

Theorem 3: Assume $\bar{v}_{1} \leq \bar{v}_{2} \leq \cdots \leq \bar{v}_{k_{\mathrm{D}}}$ and denote $\mathbf{V}^{(m)}(v)=[\underbrace{v, \cdots, v}_{m}, \bar{v}_{m+1}, \cdots, \bar{v}_{k_{\mathrm{D}}}]$, we have $\mathbf{V}^{(m)}\left(\bar{v}_{m}\right)=[\underbrace{\bar{v}_{m}, \cdots, \bar{v}_{m}}_{m}, \bar{v}_{m+1}, \cdots, \bar{v}_{k_{\mathrm{D}}}]$ and $\mathbf{P}\left(\mathbf{V}^{(m)}\left(\bar{v}_{m}\right)\right)=H^{-1}\left(\mathbf{V}^{(m)}\left(\bar{v}_{m}\right)\right) \mathbf{N}$, where $H\left(\mathbf{V}^{(m)}\left(\bar{v}_{m}\right)\right)$ is given in (20) and $\mathbf{N}=\left[N_{1}, N_{2}, \cdots, N_{k_{\mathrm{D}}}\right]^{\mathrm{T}}$.

- For $1 \leq m<k_{\mathrm{D}}$, if $\overline{\mathbf{P}} \preceq \mathbf{P}\left(\mathbf{V}^{(m)}\left(\bar{v}_{m}\right)\right) \preceq \mathbf{P}^{\max }$, where $\mathbf{P}^{\max }=\mathbf{P}=\left[p_{1}^{\max }, p_{2}^{\max }, \cdots, p_{k_{\mathrm{D}}}^{\max }\right]^{\mathrm{T}}$, holds and $\overline{\mathbf{P}} \preceq \mathbf{P}\left(\mathbf{V}^{(m)}\left(\bar{v}_{m+1}\right)\right) \preceq \mathbf{P}^{\max }$ does not hold, the optimal power allocation in problem $\left(\mathrm{P}_{3}\right)$ enables the optimal SINR vector $\mathbf{V}^{*}=\left[v_{1}^{*}, v_{2}^{*}, \cdots, v_{k_{\mathrm{D}}}^{*}\right]$ to satisfy $\mathbf{V}^{*}=$ $\mathbf{V}^{(m)}\left(v^{*}\right)=[\underbrace{v^{*}, \cdots, v^{*}}_{m}, \bar{v}_{m+1}, \cdots, \bar{v}_{k_{\mathrm{D}}}]$, where $\bar{v}_{m} \leq v^{*}<\bar{v}_{m+1}$.

- For $m=k_{\mathrm{D}}$, if $\overline{\mathbf{P}}^{m} \preceq \mathbf{P}\left(\mathbf{V}^{\left(k_{\mathrm{D}}\right)}\left(\bar{v}_{k_{\mathrm{D}}}\right)\right) \preceq \mathbf{P}^{\max }$ holds, the optimal power allocation in problem $\left(\mathrm{P}_{3}\right)$ enables the optimal SINR vector $\mathbf{V}^{*}=\left[v_{1}^{*}, v_{2}^{*}, \cdots, v_{k_{\mathrm{D}}}^{*}\right]$ to satisfy $\mathbf{V}^{*}=\mathbf{V}^{(m)}\left(v^{*}\right)=$ 


\section{$[\underbrace{v^{*}, \cdots, v^{*}}_{k_{\mathrm{D}}}]$, where $v^{*} \geq \bar{v}_{k_{\mathrm{D}}}$.}

Proof: The proof is provided in Appendix C.

Suppose that there exists a user $m\left(1 \leq m<k_{\mathrm{D}}\right)$, which satisfies $\overline{\mathbf{P}} \preceq \mathbf{P}\left(\mathbf{V}^{(m)}\left(\bar{v}_{m}\right)\right) \preceq \mathbf{P}^{\max }$ but does not satisfy $\overline{\mathbf{P}} \preceq \mathbf{P}\left(\mathbf{V}^{(m)}\left(\bar{v}_{m+1}\right)\right) \preceq \mathbf{P}^{\max }$. From Theorem 3, the optimal power allocation $\mathbf{P}^{*}$ in problem $\left(\mathbf{P}_{3}\right)$ enables $\mathbf{V}^{*}=[\underbrace{v^{*}, \cdots, v^{*}}_{m}, \bar{v}_{m+1}, \cdots, v_{k_{\mathrm{D}}}]$ and can be written as

$$
\mathbf{P}^{*}=H^{-1}\left(\mathbf{V}^{*}\right) \mathbf{N}
$$

which means that the optimal power allocation $\mathbf{P}^{*}$ can be obtained by calculating $H^{-1}\left(\mathbf{V}^{*}\right) \mathbf{N}$. However, the optimal SINR vector $\mathbf{V}^{*}$ is unknown to the BS. This makes it difficult to obtain $\mathbf{P}^{*}$ directly. Alternatively, we develop a binary-search based algorithm to approach $\mathbf{V}^{*}$ and then obtain $\mathbf{P}^{*}$. Specifically, the optimal SINR vector is $\mathbf{V}^{*}=\mathbf{V}^{(m)}\left(v^{*}\right)=[\underbrace{v^{*}, \cdots, v^{*}}_{m}, \bar{v}_{m+1}, \cdots, v_{k_{\mathrm{D}}}]$, where $\bar{v}_{m} \leq v^{*}<\bar{v}_{m+1}$. Consider that each transmit power in $\mathbf{P}^{*}$ increases if any SINR in $\mathbf{V}^{*}$ increases from Theorem 1. Then, we may approach $\mathbf{V}^{*}$ by applying binary search between $\mathbf{V}^{(m)}\left(\bar{v}_{m}\right)$ and $\mathbf{V}^{(m)}\left(\bar{v}_{m+1}\right)$ subject to the transmit power constraints.

Similarly, if $\overline{\mathbf{P}} \preceq H^{-1}\left(\mathbf{V}^{\left(k_{\mathrm{D}}\right)}\left(\bar{V}_{k_{\mathrm{D}}}\right)\right) \mathbf{N} \preceq \mathbf{P}^{\max }$ holds, the optimal SINRs can be denoted as $\mathbf{V}^{*}=\mathbf{V}^{\left(k_{\mathrm{D}}\right)}\left(v_{k_{\mathrm{D}}}\right)=[\underbrace{v^{*}, \cdots, v^{*}}_{k_{\mathrm{D}}}]$, where $v^{*} \geq \bar{v}_{k_{\mathrm{D}}}$. Meanwhile, consider that the optimal SINR vector $v^{*}$ is upper-bounded by $\min _{m \in \mathcal{S}_{\mathrm{D}}^{*}} \frac{p_{m}^{\max } g(m, m)}{N_{m}}$, we have $\bar{v}_{k_{\mathrm{D}}} \leq v^{*} \leq \min _{m \in \mathcal{S}_{\mathrm{D}}^{*}} \frac{p_{m}^{\max } g(m, m)}{N_{m}}$. Then, we may approach $\mathbf{V}^{*}$ by applying binary search between $\mathbf{V}^{\left(k_{\mathrm{D}}\right)}\left(\bar{v}_{k_{\mathrm{D}}}\right)$ and $\mathbf{V}^{\left(k_{\mathrm{D}}\right)}\left(\min _{m \in \mathcal{S}_{\mathrm{D}}^{*}} \frac{p_{m}^{\max } g(m, m)}{N_{m}}\right)$ subject to transmit power constraints. To summarize, we illustrate the binary-search based power allocation in Algorithm 3 .

\section{B. Complexity Analysis}

The computational complexity of Algorithm 3 is dominated by two loops. In what follows, we will analyze the computational complexity of the two loop, respectively.

For the first loop from Line 1 to Line 6 , we consider the worst case that there are $k_{\mathrm{D}}$ rounds. In each round, the computational complexity is dominated by the calculation of $\mathbf{P}\left(\Gamma^{(m)}\left(\bar{v}_{m+1}\right)\right)=$ $H^{-1}\left(\mathbf{V}^{(m)}\left(\bar{v}_{m+1}\right)\right) \mathbf{N}$, which consists of a matrix inversion operation and a matrix multiplication operation. Since the computational complexity of the inversion of a $k_{\mathrm{D}} \times k_{\mathrm{D}}$ matrix $H\left(\mathbf{V}^{(m)}\left(\bar{v}_{m+1}\right)\right)$ is $O\left(k_{\mathrm{D}}^{2.373}\right)$ and that of the multiplication of a $k_{\mathrm{D}} \times k_{\mathrm{D}}$ matrix $H^{-1}\left(\mathbf{V}^{(m)}\left(\bar{v}_{m+1}\right)\right)$ 


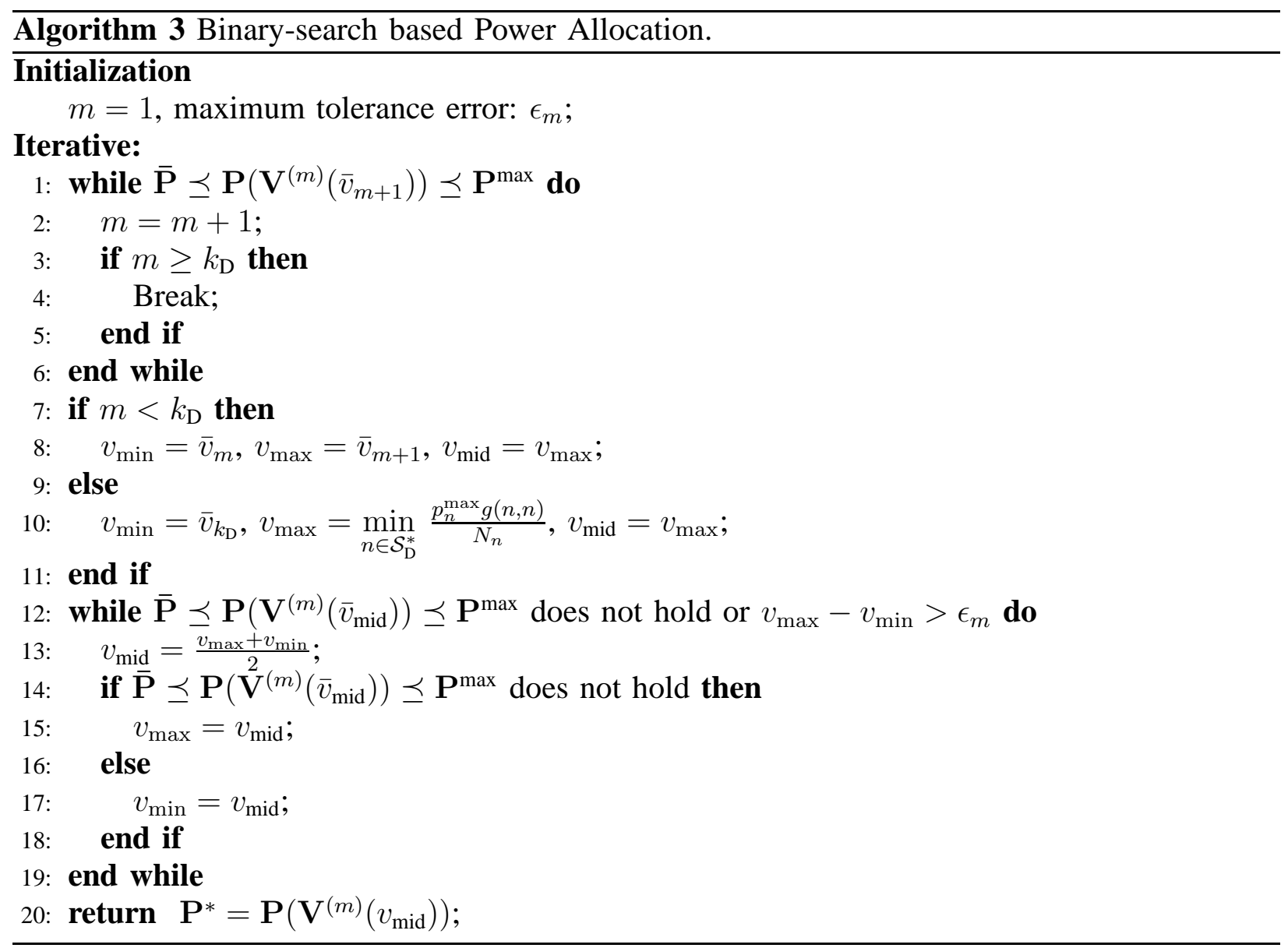

and a $k_{\mathrm{D}} \times 1$ vector $\mathbf{N}$ is a $O\left(k_{\mathrm{D}}^{2}\right)$ [37], we have the computational complexity to derive $\mathbf{P}\left(\mathbf{V}^{(m)}\left(\bar{v}_{m+1}\right)\right)$ is $O\left(k_{\mathrm{D}}^{2.373}\right)+O\left(k_{\mathrm{D}}^{2}\right)=O\left(k_{\mathrm{D}}^{2.373}\right)$. Then, the computational complexity of the first loop is $O\left(k_{\mathrm{D}}^{3.373}\right)$.

For the second loop from Line 12 to Line 19, the round of the binary search is $O(\log \phi)$ [38], where $\phi=\frac{\min _{m \in \mathcal{S}_{\mathrm{D}}^{*}} \frac{p_{m}^{\max } g(m, m)}{N_{m}}-\bar{v}_{k_{\mathrm{D}}}}{e_{m}}$. In each round, the computational complexity is dominated by the calculation of $\mathbf{P}\left(\mathbf{V}^{(m)}\left(\bar{v}_{\text {mid }}\right)\right)$ and thus is $O\left(k_{\mathrm{D}}^{2.373}\right)$. Then, the computational complexity of the second loop is $O\left(k_{\mathrm{D}}^{2.373} \log \phi\right)$.

Consequently, the computational complexity of Algorithm 3 is $O\left(k_{\mathrm{D}}^{3.373}\right)+O\left(k_{\mathrm{D}}^{2.373} \log \phi\right)$.

\section{NUMERICAL RESULTS}

In this section, we will show the performance of our proposed algorithms from numerical sides. To show the advantages of the proposed algorithms, we will also give the performance 


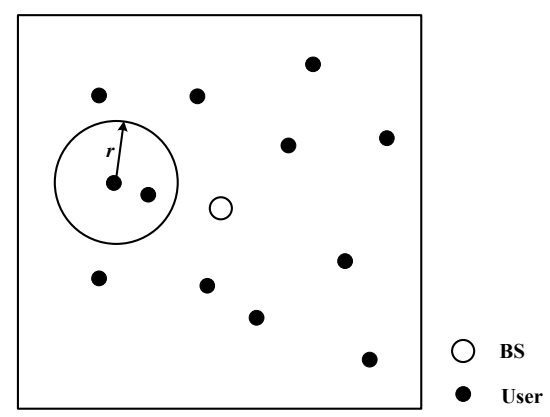

Figure 2. Simulation network model, where the empty circle denotes the BS and a solid circle denotes a user.

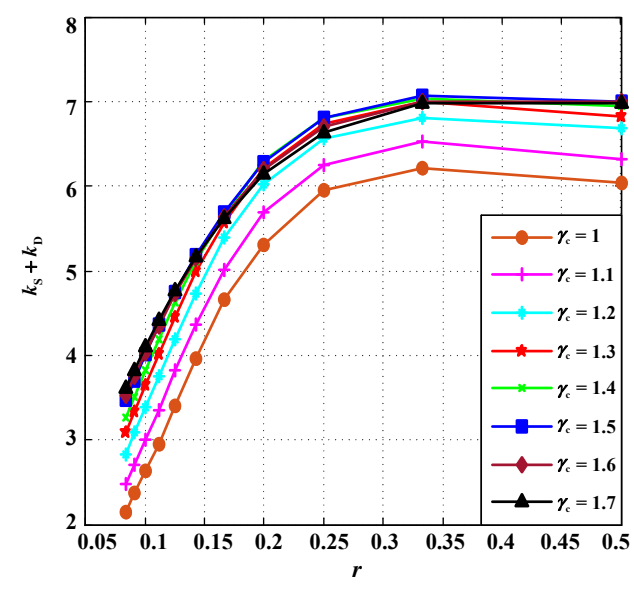

Figure 3. The number of users that can obtain the requested files from their own memory or from helpers through D2D transmissions, i.e., $k_{\mathrm{S}}+k_{\mathrm{D}}$, with different help distances $r$ and file caching coefficients $\gamma_{c}$, where $v_{\mathrm{T}}=0 \mathrm{~dB}, c_{\mathrm{s}}=0 \mathrm{~dB}$, $\gamma_{r}=0.6$, and $K=100$.

comparison with the existing similar algorithms in [25], [26], [32], and [34].

In our simulation, we adopt the pathloss channel as $y=\frac{\lambda}{4 \pi d} x+n_{0}$, where $y$ is the received signal at the receiver, $x$ is the transmit signal at the transmitter, $d$ is the distance between two transceivers, $\lambda$ is the wavelength of the carrier, and $n_{0}$ is the AWGN with power spectral density $N_{0}=-170 \mathrm{dBm} / \mathrm{Hz}$ at the receiver. We assume that all the maximum transmit power constraints are the same, i.e., $p_{m}^{\max }=20 \mathrm{dBm}, \forall 1 \leq m \leq K$, and that the minimum acceptable SINRs at all the scheduled D2D receivers are the same, i.e., $\check{v}_{m}=v_{\mathrm{T}}, \forall m \in \mathcal{S}_{\mathrm{D}}^{*}$, that the number of overall files is $N=1000$, and the bandwidth for D2D communication is $1 \mathrm{MHz}$. For fair comparison with [26], we assume a square cell with each side $1 \mathrm{~km}$ as shown in Fig. 2.

Fig. 3 provides the number of users that can obtain the requested file from their own memory or from helpers through $\mathrm{D} 2 \mathrm{D}$ transmissions, i.e., $k_{\mathrm{S}}+k_{\mathrm{D}}$, with different help distances $r$ and 


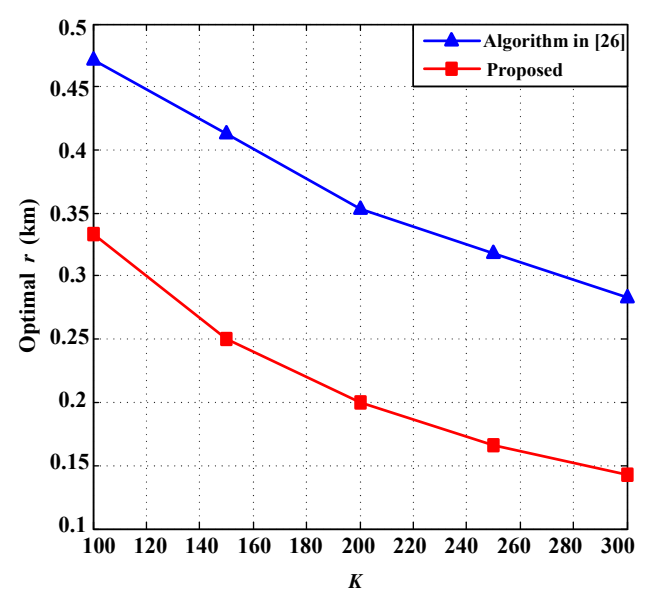

Figure 4. The Optimal help distance versus different number of users in the cell, where $v_{\mathrm{T}}=0 \mathrm{~dB}, c_{\mathrm{s}}=0 \mathrm{~dB}, \gamma_{r}=0.6$.

file caching coefficients $\gamma_{c}$. From this figure, the $k_{\mathrm{S}}+k_{\mathrm{D}}$ first increases and then decreases as $r$ grows. In fact, although $k_{\mathrm{S}}$ is only determined by the caching coefficient, $k_{\mathrm{D}}$ is directly affected by two factors. One is the number of the potential D2D links $k_{\mathrm{DB}}$. The other one is the interference among the scheduled D2D links. Then, $k_{\mathrm{S}}+k_{\mathrm{D}}$ is more sensitive to the variation of $k_{\mathrm{D}}$ compared with $k_{\mathrm{S}}$. As $r$ grows, the chance that one user finds the requested file from other users memory increases. This increases $k_{\mathrm{DB}}, k_{\mathrm{D}}$ and $k_{\mathrm{S}}+k_{\mathrm{D}}$. As $r$ continues to grow, the average distance between D2D transceivers increases. This leads to higher transmit power at each D2D transmitter. Thus, the mutual interference among the scheduled D2D links increases, and decreases $k_{\mathrm{S}}+k_{\mathrm{D}}$. Besides, we observe that the optimal $r$ and $\gamma_{c}$ in terms of the largest $k_{\mathrm{S}}+k_{\mathrm{D}}$ is about $0.33 \mathrm{~km}$ and 1.5 , respectively.

Fig. 4 gives the optimal $r$ with different number of users $K$. For comparison, we also provide the optimal $r$ in [26]. It is clear that the optimal $r$ decreases as $K$ grows. For small $K$, the number of the potential D2D links $k_{\mathrm{DB}}$ limits the number of users that can obtain the requested file from their own memory or from helpers through D2D transmissions, i.e., $k_{\mathrm{S}}+k_{\mathrm{D}}$. To maximize $k_{\mathrm{S}}+k_{\mathrm{D}}$, the optimal $r$ needs to be large to increase the potential D2D links. As $K$ grows, a small $r$ may result in a large number of the potential D2D links $k_{\mathrm{DB}}$, which generates strong mutual interference to each other and limits the number of the scheduled D2D links. Thus, a smaller optimal $r$ is needed for a larger $K$. Besides, we observe that the optimal $r$ in our algorithm is smaller than the optimal $r$ in [26]. This is reasonable since each user in our algorithm can establish more potential D2D links than the algorithm in [26] for a given number of users. Then, 


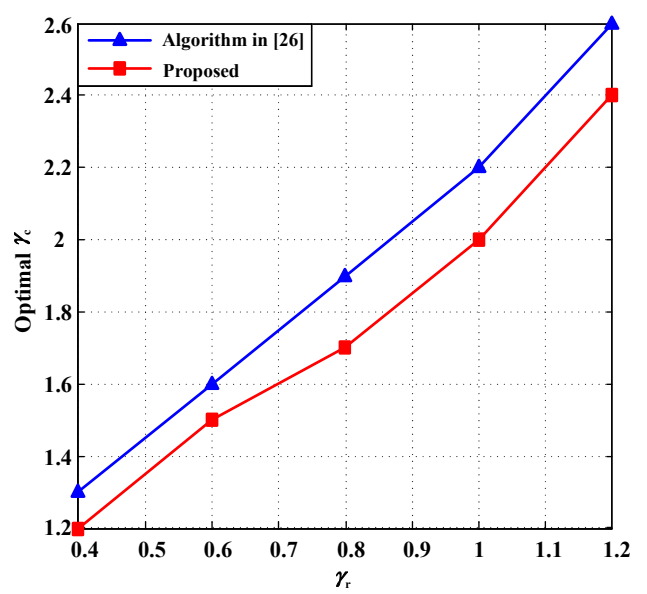

Figure 5. The Optimal file caching coefficient versus different file request coefficients, where $v_{\mathrm{T}}=0 \mathrm{~dB}, c_{\mathrm{s}}=0 \mathrm{~dB}, K=100$.

a smaller optimal $r$ is needed to maximize $k_{\mathrm{S}}+k_{\mathrm{D}}$.

Fig. 5 gives the optimal $\gamma_{c}$ with different file request coefficients $\gamma_{r}$. For comparison, we also provide the optimal $\gamma_{c}$ in [26]. It is observed that, the optimal $r$ increases as $\gamma_{c}$ grows. When $\gamma_{r}$ is small, more different files are requested by the users in the cell. Then, users need to cache more different files to maximize the number of the scheduled D2D links. Thus, the optimal $\gamma_{c}$ is small. Otherwise, a big optimal $\gamma_{c}$ is required. Besides, we observe that the optimal $\gamma_{c}$ in our algorithm is smaller than the optimal $\gamma_{c}$ in [26]. This is because each user in our algorithm can potentially help more users than that in [26]. Then, users in our algorithm is required to cache more different files for D2D transmissions. Thus, a smaller optimal $\gamma_{c}$ is needed in our algorithm.

Fig. 6 compares the number of the scheduled D2D links of the proposed CPC-based D2D link scheduling algorithm with the DCPC-based D2D link scheduling algorithm in [32] and the optimal schedule algorithm from the exhaustive search. Since the computational complexity of the exhaustive search with a large number of potential D2D links $k_{\mathrm{DB}}$ is too high, we limit the number of the potential D2D links by choosing a small help distance instead of the optimal one. It could be concluded from this figure that, the performance of the proposed algorithm is between the performance of the optimal schedule algorithm and the DCPC-based schedule algorithm in [32]. This indicates that the CPC-based D2D link scheduling algorithm is more flexible than that the DCPC-based D2D link scheduling algorithm in [32].

Fig. 7 shows the system throughput of the scheduled D2D links with the binary-search 


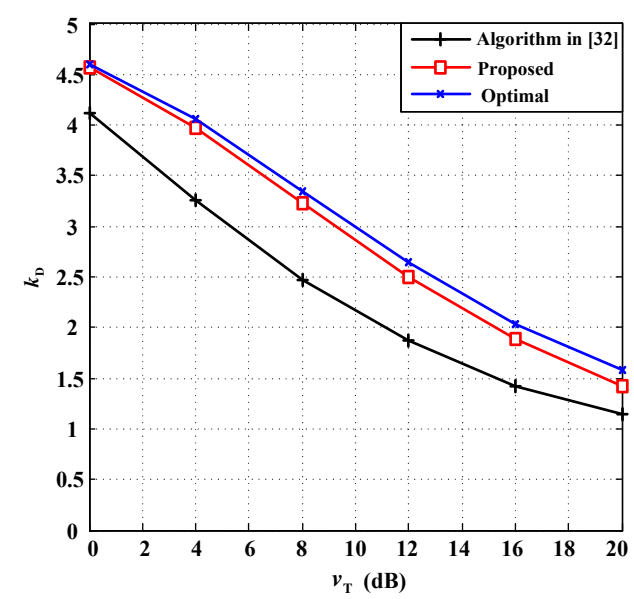

Figure 6. Comparison of the numbers of the scheduled D2D links with different scheduling algorithms, where $v_{\mathrm{T}}=0 \mathrm{~dB}$, $c_{\mathrm{s}}=0 \mathrm{~dB}, \gamma_{r}=0.6, \gamma_{c}=1.5, r=\frac{1}{7} \mathrm{~km}$.

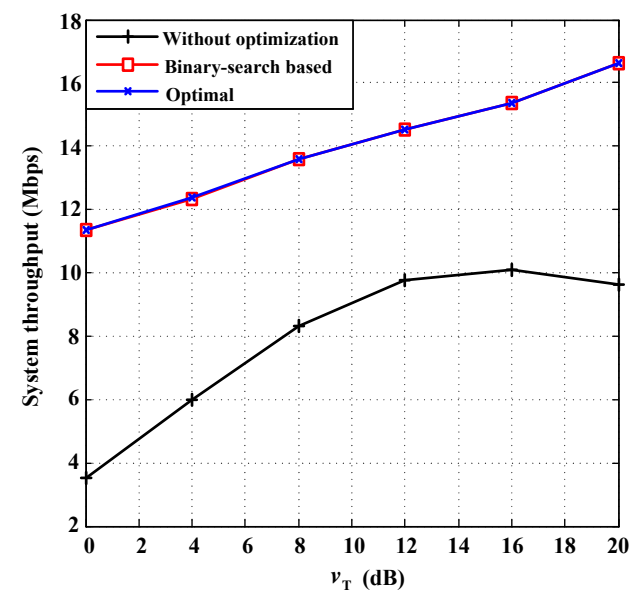

Figure 7. Comparison of the system throughput with different power allocation algorithms, where $c_{\mathrm{s}}=v_{\mathrm{T}}, K=100, \gamma_{r}=0.6$, $\gamma_{c}=1.5$, and $r=\frac{1}{7} \mathrm{~km}$.

based power allocation algorithm in Algorithm 3. Here, the system throughput is calculated by $\sum_{m \in \mathcal{S}_{\mathrm{D}}^{*}} \log \left(v_{m}+1\right)$. For comparison, we provide the performance of the optimal power allocation algorithm, where a convex optimization toolbox is adopted, and the algorithm without optimization, where each D2D link works with the minimum SINR. From Fig. 7 the performance curve of the proposed binary-search based power allocation algorithm almost overlaps with the optimal one. This validates our analysis and indicates that our proposed algorithm may achieve similar rate performance with the optimal algorithm and outperforms the algorithm without optimized power allocation, i.e., more than $40 \%$ improvement. It should be noted that the proposed optimal 

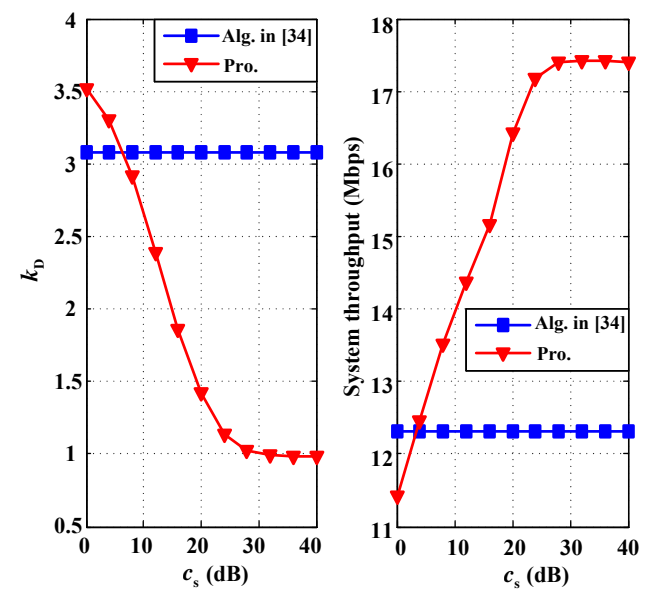

Figure 8. Performance comparison with scheduling algorithm in [34] for different scheduling coefficient $c_{\mathrm{s}}$, where $v_{\mathrm{T}}=0 \mathrm{~dB}$, $K=100, \gamma_{c}=1.5$ and $r=\frac{1}{7} \mathrm{~km}$.

power allocation algorithm is based on max-min fairness, which achieves perfect fairness. In fact, the system throughput can be further improved if some unfairness can be tolerated [32].

Fig. 8 shows the number of the D2D links and the system throughput with different scheduling coefficient $c_{\mathrm{s}}$. We observe that the number of the D2D links decreases as $c_{\mathrm{S}}$ grows. This coincides with the intuition that a larger $c_{\mathrm{s}}$ means a stricter condition on the scheduled D2D links. Then, the D2D links with stronger communication channels and weaker interference channels are scheduled. On the other hand, the system throughput increases as $c_{\mathrm{s}}$ grows. This is quite reasonable since fewer D2D links mean less mutual interference, which boosts the system throughput. On the other hand, the system throughput of the optimal scheduling in terms of the largest number of the scheduled D2D links is much smaller than the largest system throughput. Thus, the number of the scheduled D2D links and the system throughput can be balanced by choosing a proper $c_{\mathrm{s}}$.

Meanwhile, we provide the performance of the algorithm in [34], where only the information theoretic independent sets are scheduled. Note that there is no power allocation in [34], we apply Algorithm 3 in [34] for fair comparison. From the figure, the number of the D2D links and the system throughput are constant since the scheduling algorithm in [34] is not affected by $c_{\mathrm{s}}$. We also observe that the number of the scheduled D2D links and the system throughput with the proposed algorithms can simultaneously outperform those with the algorithm in [34]. For instance, $c_{\mathrm{s}}$ should be chosen around between four and six in this figure, e.g., $c_{\mathrm{s}}=5 \mathrm{~dB}$. In this 

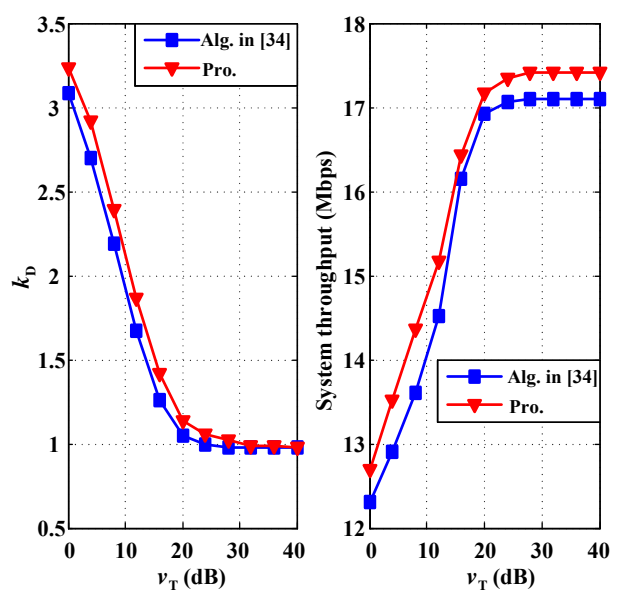

Figure 9. Performance comparison with the scheduling algorithm in [34] in terms of $k_{\mathrm{D}}$ and system throughput for different $v_{\mathrm{T}}$, where $K=100, \gamma_{c}=1.5$ and $r=\frac{1}{7} \mathrm{~km}$.

way, we may choose proper values of $c_{\mathrm{s}}$ for different system parameters.

\begin{tabular}{|c|c|c|c|c|c|c|c|c|c|c|c|}
\hline$v_{\mathrm{T}}(\mathrm{dB})$ & 0 & 4 & 8 & 12 & 16 & 20 & 24 & 28 & 32 & 36 & 40 \\
\hline$c_{\mathrm{s}}(\mathrm{dB})$ & 5 & 8 & 12 & 16 & 20 & 24 & 26 & 28 & 32 & 36 & 40 \\
\hline
\end{tabular}

Table I

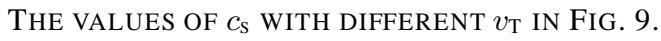

Fig. 9 compares the proposed algorithm with the algorithms in [34] in terms of the number of the scheduled D2D links and the system throughput. With the same selection method of $c_{\mathrm{s}}$ in Fig. 8, we choose the values of $c_{\mathrm{s}}$ with different $v_{\mathrm{T}}$ in Tab. I. It is observed that the proposed algorithms outperform the algorithm in [34] in terms of both the number of the scheduled D2D links and the system throughput.

Fig. 10 compares the proposed algorithm (Pro. in the figure) with algorithm A (Alg. A in the figure) and algorithm B (Alg. B in the figure) in terms of the number of the scheduled D2D links and system throughput. In this figure, (I) and (II) denote $k_{\mathrm{D}}$ and $k_{\mathrm{S}}+k_{\mathrm{D}}$, respectively. In algorithm A, we adopt the Zipf-distribution caching and scheduling scheme in [26], and the proposed optimal power allocation algorithm with perfect fairness meanwhile considering the minimum acceptable SINR constraint, i.e., $v_{\mathrm{T}}=0 \mathrm{~dB}$. In algorithm B, we adopt the optimal cluster-based caching and scheduling scheme in [25], and the proposed power allocation algorithm with perfect fairness meanwhile considering the minimum acceptable SINR constraint, i.e., $v_{\mathrm{T}}=0 \mathrm{~dB}$. The 

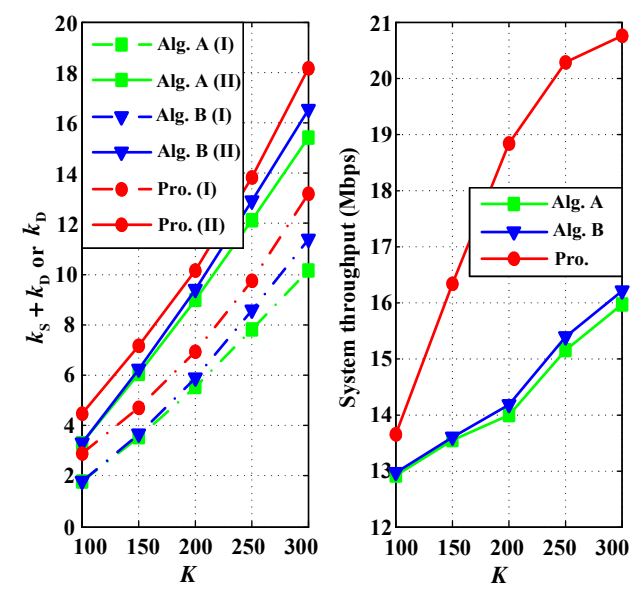

Figure 10. Performance comparison with the scheduling algorithms in [25] and [26] in terms of $k_{\mathrm{S}}+k_{\mathrm{D}}, k_{\mathrm{D}}$, and system throughput for different number of users in the cell, where $v_{\mathrm{T}}=0 \mathrm{~dB}, \gamma_{r}=0.6, \gamma_{c}=1.5, r=\frac{1}{7} \mathrm{~km}$.

scheduling coefficient $c_{\mathrm{s}}$ of the proposed algorithm is obtained with the same selection method in Fig. 8 for each $K$. That is, $c_{\mathrm{s}}$ is chosen to be $10 \mathrm{~dB}, 8 \mathrm{~dB}, 6 \mathrm{~dB}, 4 \mathrm{~dB}$, and $2 \mathrm{~dB}$ when $K$ is 100, 150, 200, 250, and 300, respectively. From this figure, the proposed algorithms outperform the algorithms in [25] and [26] in terms of the number of the scheduled D2D links, the number of users that can obtain the requested files either from their own memory or from helpers through D2D transmissions, and the system throughput. This is intuitive since the proposed algorithms create more D2D links with strong communication channels and weak interference channels. Then, the number of the scheduled D2D links and the system throughput can be simultaneously enhanced by efficiently scheduling and power allocation.

Fig. 11 compares the three algorithms (Alg. A, Alg. B, and Pro. in Fig. 10) in terms of the download time, which is calculated by $\sum_{m \in S_{\mathrm{B}} \cup S_{\mathrm{DB}} \backslash S_{\mathrm{D}}^{*}} w_{m}^{\mathrm{BS}}+\sum_{m \in S_{\mathrm{D}}^{*}} w_{m}^{\mathrm{D} 2 \mathrm{D}}$ [26], where $w_{m}^{\mathrm{BS}}$ is the download time of a video file from the $\mathrm{BS}$ to user $m$ and $w_{m}^{\mathrm{D} 2 \mathrm{D}}$ is the download time of a video file from a helper to user $m$ through an one-hop D2D transmission. Specifically, $w_{m}^{\mathrm{BS}}$ is calculated by $w_{m}^{\mathrm{BS}}=\frac{L_{\mathrm{video}}}{R_{m}^{\mathrm{BS}}}$, where $L_{\text {video }}$ is the length of a file, say $L_{\text {video }}=30 \mathrm{MB}$ in the simulation, and $R_{m}^{\mathrm{BS}}$ is the transmission rate from the BS to user $m$ and is assumed to be $R_{m}^{\mathrm{BS}}=120 \mathrm{kbps}$ [26], $\forall m \in S_{\mathrm{B}} \cup S_{\mathrm{DB}} \backslash S_{\mathrm{D}}^{*}$. Similarly, $w_{m}^{\mathrm{D} 2 \mathrm{D}}$ is calculated by $w_{m}^{\mathrm{D} 2 \mathrm{D}}=\frac{L_{\text {video }}}{R_{m}^{\mathrm{D} 2 \mathrm{D}}}$, where $R_{m}^{\mathrm{D} 2 \mathrm{D}}$ is the transmission rate of $\mathrm{D} 2 \mathrm{D}$ link $l_{m}$ and is assumed to be $R_{m}^{\mathrm{D} 2 \mathrm{D}}=\log \left(v_{m}+1\right) \mathrm{Mbps}$. From this figure, the proposed algorithms outperforms both Alg. A and Alg. B. This is reasonable since more users in the proposed algorithms can obtain the requested files from their own memory or 


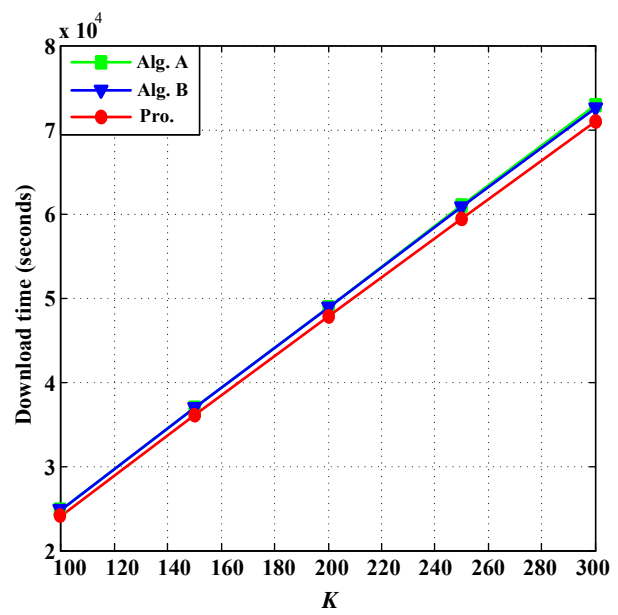

Figure 11. Performance comparison with the scheduling algorithm in [26] in terms of the download time for different number of users in the cell, where $v_{\mathrm{T}}=0 \mathrm{~dB}, \gamma_{r}=0.6, \gamma_{c}=1.5, r=\frac{1}{7} \mathrm{~km}$.

from helpers through D2D transmissions compared with those in either Alg. A or Alg. B (as shown in Fig. 10). Thus, the average download time is reduced with the proposed algorithms.

\section{CONCLUSIONS AND Future Discussions}

We have studied the efficient scheduling and power allocation of D2D-assisted wireless caching networks. We formulate a joint D2D link scheduling and power allocation problem to maximize the system throughput. However, the problem is non-convex and obtaining the optimal solution is computationally hard. Alternatively, we seek to obtain a suboptimal solution with reasonable complexity. Briefly, we intend to schedule the D2D links with strong communication channels and weak interference channels and allocate the power to the scheduled D2D links fairly. Thus, we decompose the system throughput maximization problem into a D2D link scheduling problem and an optimal power allocation problem. To solve the two subproblems, we first develop a D2D link scheduling algorithm to increase the number of the scheduled D2D links satisfying both the SINR and the transmit power constraints. Then, we develop an optimal power allocation algorithm to maximize the minimum transmission rate of the scheduled D2D links. Numerical results indicate that both the number of the scheduled D2D links and the system throughput can be improved simultaneously with the Zipf-distribution caching scheme, the proposed D2D link scheduling algorithm, and the proposed optimal power allocation algorithm compared with the state of arts. 
From the results in this paper, we also conclude that the D2D communication in a wireless caching network can be enhanced by creating more D2D links with strong communication channels and weak interference channels. This can be achieved by optimized caching, the proposed D2D links scheduling algorithm, and the proposed optimal power allocation algorithm. Although the optimized Zipf-distribution caching scheme in this paper is not generally optimal, the proposed D2D link scheduling algorithm and the proposed optimal power allocation algorithm can be used for any caching scheme. Ideally, the joint caching, scheduling, and power allocation algorithm should be able to further improve D2D communication. However, even the optimal caching distribution of the general model in our paper is quite hard. A further study on the optimal caching scheme is beyond the scope of this paper.

Throughout this paper, we assume one channel to simplify the illustration of our algorithms. In fact, the proposed D2D link scheduling algorithm and the proposed optimal power allocation algorithm can be used in multi-channel systems, e.g., FDMA and TDMA. More specifically, D2D links are iteratively scheduled in a unique channel, i.e., a frequency band (corresponding to FDMA) or a time slot (corresponding to TDMA). This process terminates in two cases. One is that there is no more channels to accommodate the scheduled D2D links. The other one is that all the D2D links have been scheduled. Since more wireless resource is used in multi-channel systems, a better system performance is expected.

Besides, both the proposed D2D link scheduling algorithm and the proposed optimal power allocation algorithm are achieved in a centralized manner. That is, all the calculations are conducted at the BS. This requires the BS to keep track of the cached content in each memory and collect the channel state information among different potential D2D links. One direction of the future work is to develop decentralized algorithms of scheduling and power allocation in the D2D assisted wireless caching networks.

\section{APPENDIX}

\section{A. Proof of Theorem 1}

To prove Theorem 1, we will first prove part I: each transmit power $p_{n}, \forall n \in \mathcal{S}_{\mathrm{D}}$, increases if any SINR $v_{m}, m \in \mathcal{S}_{\mathrm{D}}$, in $\left(\mathrm{P}_{1}\right)$ increases. Then, we will prove part II: the value of $\left|\mathcal{S}_{\mathrm{D}}\right|$ remains constant or decreases if any $\operatorname{SINR} v_{m}, m \in \mathcal{S}_{\mathrm{D}}$, in $\left(\mathrm{P}_{1}\right)$ increases. 
1) Proof of part I in Theorem 1: Suppose that there is a power increment $\Delta p_{m}$ and a SINR increment $\Delta v_{m}$ for D2D link $l_{m}$ in $\mathcal{S}_{\mathrm{D}}$ such that

$$
v_{m}+\Delta v_{m}=\frac{\left(p_{m}+\Delta p_{m}\right) g(m, m)}{\sum_{n \in \mathcal{S}_{\mathrm{D}}, n \neq m} p_{n} g(n, m)+N_{m}} .
$$

Then, there must be a SINR decrement $\Delta v_{n}$ for any other D2D link $l_{n}\left(\forall n \in \mathcal{S}_{\mathrm{D}}, n \neq m\right)$ such that

$$
v_{n}-\Delta v_{n}=\frac{p_{n} g(n, n)}{\sum_{k \in \mathcal{S}_{\mathrm{D}}, k \neq n, k \neq m} p_{k} g(k, n)+\left(p_{m}+\Delta p_{m}\right) g(m, n)+N_{n}} .
$$

To remain the SINR $v_{n}$ at D2D link $l_{n}, p_{n}\left(\forall n \in \mathcal{S}_{\mathrm{D}}, n \neq m\right)$ will be increased by $\Delta p_{n}$, such that

$$
v_{n}=\frac{\left(p_{n}+\Delta p_{n}\right) g(n, n)}{\sum_{k \in \mathcal{S}_{\mathrm{D}}, k \neq n}\left(p_{k}+\Delta p_{k}\right) g(k, n)+N_{n}} .
$$

Thus, each $p_{n}, n \in \mathcal{S}_{\mathrm{D}}$ increases if there is an increment of any $v_{m}, m \in \mathcal{S}_{\mathrm{D}}$.

2) Proof of part II in Theorem 1: Suppose that the D2D links in $\mathcal{L}_{\mathrm{D}}$ satisfy all the constraints in problem $\left(\mathbf{P}_{1}\right)$ with transmit power $\mathbf{P}=\left\{p_{1}, p_{2}, \cdots, p_{k_{\mathrm{D}}}\right\}$. If there is a SINR increment $\Delta v_{m}$ at D2D link $l_{m}$, the transmit power should be increased to $\mathbf{P}+\Delta \mathbf{P}=\left\{p_{1}+\Delta p_{1}, p_{2}+\Delta p_{2}, \cdots, p_{k_{\mathrm{D}}}+\right.$ $\left.\Delta p_{k_{\mathrm{D}}}\right\}$ to satisfy all the minimum acceptable SINRs. If $p_{n}+\Delta p_{n} \leq p_{n}^{\max }, \forall n \in \mathcal{S}_{\mathrm{D}}$, all the D2D links in $\mathcal{L}_{\mathrm{D}}$ can still be satisfied with the minimum SINR constraints and number of the scheduled D2D links remains. If there exists a $\Delta p_{n}\left(n \in \mathcal{S}_{\mathrm{D}}\right)$ satisfying $\bar{p}_{n}+\Delta p_{n}>p_{n}^{\max }$ and

$$
v_{n}=\frac{p_{n}^{\max } g(n, n)}{\sum_{k \in \mathcal{S}_{\mathrm{D}}, k \neq n}\left(p_{k}+\Delta p_{k}\right) g(k, n)+N_{n}}<\bar{v}_{n} .
$$

Then, the D2D link $l_{n}$ cannot be satisfied with the minimum SINR constraint. This reduces the number of the scheduled D2D transmissions. This completes the proof of Theorem 1.

\section{B. Proof of Theorem 2}

The regular admission control problem with QoS constraint has been proved to be NP-hard in [32] and is a special case (Case II) of problem $\left(\mathrm{P}_{2}\right)$. Specifically, the problem $\left(\mathrm{P}_{2}\right)$ reduces to a regular admission control problem when any two D2D links in $\mathcal{L}_{\mathrm{D}}$ do not share the same users. Thus, problem $\left(\mathrm{P}_{2}\right)$ is also NP-hard. 


\section{Proof of Theorem 3}

In this part, we will prove Theorem 3. Firstly, we will give the proof of the first case that there is a user $m\left(0<m<k_{\mathrm{D}}\right)$ satisfying $\overline{\mathbf{P}} \preceq \mathbf{P}\left(\mathbf{V}^{(m)}\left(\bar{v}_{m}\right)\right) \preceq \mathbf{P}^{\max }$ but not satisfying $\overline{\mathbf{P}} \preceq \mathbf{P}\left(\mathbf{V}^{(m)}\left(\bar{v}_{m+1}\right)\right) \preceq \mathbf{P}^{\max }$, and prove that the optimal SINRs of the scheduled D2D links is $\mathbf{V}^{*}=[\underbrace{v^{*}, \cdots, v^{*}}_{m}, \bar{v}_{m+1}, \cdots, \bar{v}_{k_{\mathrm{D}}}]$, where $\bar{v}_{m} \leq v^{*} \leq \bar{v}_{m+1}$. Then, we will provide the proof of the second second case that if $\overline{\mathbf{P}} \preceq \mathbf{P}\left(\mathbf{V}^{\left(k_{\mathrm{D}}\right)}\left(\bar{v}_{k_{\mathrm{D}}}\right)\right) \preceq \mathbf{P}^{\max }$ holds, the optimal SINRs of the scheduled D2D links is $\mathbf{V}^{*}=[\underbrace{v^{*}, \cdots, v^{*}}_{k_{\mathrm{D}}}]$, where $v^{*} \geq \bar{v}_{k_{\mathrm{D}}}$.

1) Proof of the First Case: We will prove this by two steps by contradiction, the first step is to prove the first $m$ optimal SINRs are identical, i.e., $v_{n}^{*}=v^{*}$ for $1 \leq n \leq m$. The other step is to prove the last $k_{\mathrm{D}}-m$ optimal SINRs are the minimum SINR constraints, i.e., $v_{n}^{*}=\bar{v}_{n}$ for $m+1 \leq n \leq k_{\mathrm{D}}$.

Denote the optimal SINRs of the scheduled D2D links as $\mathbf{V}^{*}=[\underbrace{v_{1}^{*}, \cdots, v_{m}^{*}}_{m}, v_{m+1}^{*}, \cdots, v_{k_{\mathrm{D}}}^{*}]$. Since $\overline{\mathbf{P}} \preceq \mathbf{P}\left(\mathbf{V}^{(m)}\left(\bar{v}_{m}\right)\right) \preceq \mathbf{P}^{\max }$ holds and $\overline{\mathbf{P}} \preceq \mathbf{P}\left(\mathbf{V}^{(m)}\left(\bar{v}_{m+1}\right)\right) \preceq \mathbf{P}^{m a x}$ does not hold, we have $\bar{v}_{m} \leq v_{n}^{*}<\bar{v}_{m+1}$ for $1 \leq n \leq m$ and $v_{n}^{*} \geq \bar{v}_{n}$ for $m+1 \leq n \leq k_{\mathrm{D}}$. If we denote the optimal power allocation as $\mathbf{P}^{*}=\left[p_{1}^{*}, p_{2}^{*}, \cdots, p_{k_{\mathrm{D}}}^{*}\right]$, we have

$$
\frac{p_{n}^{*} g(n, n)}{\sum_{k=1, k \neq n}^{k_{\mathrm{D}}} p_{k}^{*} g(k, n)+N_{n}}=v_{n}^{*} \geq \bar{v}_{m} \geq \bar{v}_{n}, \forall 1 \leq n \leq m .
$$

We observe that the objective function in $\left(\mathrm{P}_{3}\right)$ is equivalent to $\min \left\{\max _{1 \leq n \leq k_{\mathrm{D}}} \frac{1}{v_{n}}\right\}$ and suppose that the optimal power allocation results in different SINRs at the first $m$ D2D links, i.e., $\max _{1 \leq n \leq m} 1 / v_{n}^{*}>$ $\min _{1 \leq n \leq m} 1 / v_{n}^{*}$, and that the D2D link $l_{n^{*}}$ has the largest SINR at first $m$ D2D links, i.e., $n^{*}=$ $\arg \min _{1 \leq n \leq m} 1 / v_{n}^{*}$, we have $v_{n^{*}}^{*}>\bar{v}_{m}$. Besides, from (26), we observe that $v_{n}^{*}$ is a strictly increasing function of $p_{n}^{*}$ and is a strictly decreasing function of $p_{k}^{*}$ for $k \neq n$. Therefore, there must be a small power decrement $\Delta p_{n^{*}}$ and SINR decrement $\Delta v_{n^{*}}$ for D2D link $l_{n^{*}}$ and a small SINR increment $\Delta v_{n}$ for other D2D links $l_{n}\left(1 \leq n \leq k_{\mathrm{D}}, n \neq n^{*}\right)$ such that the constraints in $\left(\mathrm{P}_{2}\right)$ still hold, i.e.,

$$
\frac{\left(p_{n^{*}}^{*}-\Delta p\right) g\left(n^{*}, n^{*}\right)}{\sum_{n=1, n \neq n^{*}}^{n=k_{\mathrm{D}}} p_{n}^{*} g\left(n, n^{*}\right)+N_{n^{*}}}=v_{n^{*}}^{*}-\Delta v_{n^{*}} \geq \bar{v}_{m} \geq \bar{v}_{n^{*}}
$$

and

$$
\frac{p_{n}^{*} g(n, n)}{\sum_{s=1, s \neq n^{*}, s \neq s}^{k_{\mathrm{D}}} p_{s}^{*} g(s, n)+\left(p_{n^{*}}^{*}-\Delta p_{n^{*}}\right) g\left(n^{*}, n\right)+N_{k}}=v_{n}^{*}+\Delta v_{n}>\bar{v}_{n}
$$


Suppose that we choose a small $\Delta v_{n}$ to satisfy $\frac{1}{\left(v_{n^{*}}^{*}-\Delta v_{n^{*}}\right)} \leq \max _{1 \leq n \leq m, n \neq n^{*}} \frac{1}{\left(v_{n}^{*}+\Delta v_{n}\right)}$. Then, we have

$$
\begin{aligned}
& \max _{1 \leq n \leq m}\left\{\frac{1}{v_{n}^{*}}\right\}=\max _{1 \leq n \leq m, n \neq n^{*}}\left\{\frac{1}{v_{n}^{*}}\right\}>\max _{1 \leq n \leq m, n \neq n^{*}}\left\{\frac{1}{v_{n}^{*}+\Delta v_{n}}\right\} \\
& =\max \left\{\max _{1 \leq n \leq m, n \neq n^{*}}\left\{\frac{1}{v_{n}^{*}+\Delta v_{n}}\right\}, \frac{1}{v_{n^{*}}^{*}-\Delta v_{n^{*}}}\right\} .
\end{aligned}
$$

This means that there exists another power allocation $\mathbf{P}^{\prime} \neq \mathbf{P}^{*}$ enabling the SINRs at the scheduled D2D links to be $\mathbf{V}^{\prime} \neq \mathbf{V}^{*}$ and $\max _{1 \leq n \leq m} 1 / \gamma_{n}^{*}>\max _{1 \leq n \leq m} 1 / v_{n}^{\prime}$, where $1 / v_{n}^{\prime}$ is equal to $\max _{1 \leq n \leq m, n \neq n^{*}} 1 /\left(v_{n}^{*}+\Delta v_{n}\right)$ for $n \neq n^{*}$ and is equal to $1 /\left(v_{n^{*}}^{*}-\Delta v_{n^{*}}\right)$ for $n=n^{*}$, which causes contradiction. Thus, we have $\max _{1 \leq n \leq m} 1 / v_{n}^{*}=1 / v^{*}$, where $\bar{v}_{m} \leq v^{*} \leq \bar{v}_{m+1}$.

Then, the optimal SINRs vector can be denoted as $\mathbf{V}^{*}=[\underbrace{v^{*}, \cdots, v^{*}}_{m}, v_{m+1}^{*}, \cdots, v_{k_{\mathrm{D}}}^{*}]$ and the corresponding minimum SINR is $v^{*}$. Next, we will prove $v_{n}^{*}=\bar{v}_{n}^{m}$ for $m+1 \leq n \leq k_{\mathrm{D}}$.

Suppose that there exists a user $k^{*}$ satisfying $v_{k^{*}}^{*}>\bar{v}_{k}$ for $m+1 \leq k \leq k_{\mathrm{D}}$. There must be a small power decrement $\Delta p_{k^{*}}$ and SINR decrement $\Delta v_{k^{*}}$ for D2D link $l_{k^{*}}$ and a small SINR increment $\Delta v_{k}$ for $\mathrm{D} 2 \mathrm{D}$ links $l_{k}(1 \leq k \leq m)$ such that the constraints in $\left(\mathrm{P}_{2}\right)$ still hold, i.e.,

$$
\frac{\left(p_{k^{*}}^{*}-\Delta p_{k^{*}}\right) g\left(k^{*}, k^{*}\right)}{\sum_{k=1, k \neq k^{*}}^{t=k_{\mathrm{D}}} p_{k}^{*} g\left(k, k^{*}\right)+N_{k^{*}}}=v_{k^{*}}^{*}-\Delta v_{k^{*}} \geq \bar{v}_{k^{*}}
$$

and

$$
\frac{p_{k}^{*} g(k, k)}{\sum_{t=1, t \neq k, t \neq k^{*}}^{k_{\mathrm{D}}} p_{t}^{*} g(t, k)+\left(p_{k^{*}}^{*}-\Delta p_{k^{*}}\right) g(t, k)+N_{k}}=v^{*}+\Delta v_{k} .
$$

Then, if we choose a small $\Delta p_{k^{*}}$ and $\Delta v_{k^{*}}$ to satisfy $\min _{1 \leq k \leq m} v^{*}+\Delta v_{k} \leq v_{m+1}$, the minimum SINR of the scheduled D2D links is $\min _{1 \leq k \leq m} v^{*}+\Delta v_{k}$, which causes contradiction. Thus, we have $v_{n}^{*}=\bar{v}_{n}$ for $m+1 \leq n \leq k_{\mathrm{D}}$.

This complete the proof of the first case.

2) Proof of the Second Case: The proof of the second case is similar to that of the first case and will be omitted for page limit.

\section{REFERENCES}

[1] IMT 2020 (5G) promation group, "5G Vision and Reguirements," White paper, May 2014.

[2] A. Damnjanovic, J. Montojo, Y. Wei, T. JI, T Luo, M. Vajapeyam, T. Yoo, O. Song, and D. Malladi“A survey on 3GPP heterogeneous networks," IEEE Trans. Wireless Commun., vol. 18, no. 3, pp. 10-21, Jun. 2011. 
[3] M. Garetto, P. Giaccone, E. Leonardi, "Capacity scaling in ad hoc networks with heterogeneous mobile nodes: The subcritical regime," IEEE/ACM Transactions on Networking, vol. 17, no. 6, pp. 1888-1901, Dec. 2009.

[4] H. Song, B. W. Kim, B. Mukherjee, "Long-reach optical access networks: A survey of research challenges, demonstrations, and bandwidth assignment mechanisms," IEEE Communications Surveys \& Tutorials, vol. 12, no. 1, pp. 112-123, Jan. 2010.

[5] E. Bastug, M. Bennis, and M. Debbah, "Living on the edge: The role of proactive caching in 5G wireless networks," IEEE Commun. Magazine, vol. 52, no. 8, pp. 82-89, Aug. 2014.

[6] A. F. Molisch, G. Caire, D. Ott, J. R. Foerster, D. Bethanabhotla, and M. Ji, "Caching eliminates the wireless bottleneck in video-aware wireless networks," arXiv preprint arXiv: 1405.5864, 2014.

[7] D. Feng, L. Lu, Y. Y. Wu, et al, "Device-to-device communications in cellular networks," IEEE Commun. Magazine, vol. 52, no. 4, pp. 49-55, Apr. 2014.

[8] M. A. Maddah-Ali and U. Niesen, "Fundamental Limits of Caching," IEEE Information Theory Proceedings (ISIT), 2013, pp. 1077-1081.

[9] M. A. Maddah-Ali and U. Niesen, "Decentralized Coded Caching Attains Order-Optimal Memory-Rate Tradeoff," arXiv preprint arXiv:1301.5848, 2014.

[10] R. Pedarsani, M. A. Maddah-Ali, and U. Niesen, “Online coded caching,” arXiv preprint arXiv:1311.3646, 2013.

[11] N. Karamchandani, U. Niesen, M. A. Maddah-Ali, and S. Diggavi, "Hierarchical Coded Caching," arXiv preprint arXiv:1403.7007, 2014.

[12] M. Ji, A. Tulino, J. Llorca, and G. Caire, "Caching and coded multicasting: Multiple groupcast index coding," IEEE Global Conference on Signal and Information Processing (GlobalSIP), 2014.

[13] M. Ji, A. Tulino, J. Llorca, and G. Caire,"Caching-aided coded multicasting with multiple random requests," IEEE Information Theory Workshop (ITW), 2015.

[14] U. Niesen and M. A. Maddah-Ali, “Coded Caching with Nonuniform Demands," arXiv preprint arXiv:1308.0378, 2014.

[15] J. Hachem, N. Karamchandani, and S. Diggavi, "Coded Caching for Heterogeneous Wireless Networks with Multi-level Access," arXiv preprint arXiv:1404.6560, 2014.

[16] J. Hachem, N. Karamchandani, and S. Diggavi, “ Multi-level Coded Caching,” arXiv preprint arXiv:1404.6563, 2014.

[17] M. Ji, A.M. Tulino, J. Llorca, and G. Caire, “ Order-optimal rate of caching and coded multicasting with random demands," arXiv preprint arXiv:1502.03124, 2015.

[18] S. Wang, W. Li, X. Tian, H. Liu, “Coded Caching with Heterogenous Cache Sizes," arXiv preprint arXiv:1504.01123. 2015.

[19] U. Niesen and M. A. Maddah-Ali, “Coded Caching for Delay-Sensitive Content,” arXiv preprint arXiv:1407.4489, 2014.

[20] A. Gharaibeh, A. Khreishah, I. Khalil, and J. Wu, “Asymptotically-Optimal Incentive-Based En-route Caching Scheme," arXiv preprint arXiv:1404.4639. 2014.

[21] A. Sengupta, R. Tandon, and T. C. Clancy, "Fundamental Limits of Caching With Secure Delivery," IEEE Trans. Inf. Forensics and Security, vol. 10, no. 2, pp. 355-370, Dec. 2014.

[22] D. Bethanabhotla, G. Caire, and M. J. Neely, "Adaptive Video Streaming for Wireless Networks with Multiple Users and Helpers," IEEE Trans. Commun., vol. 63, no. 1, pp. 268-285, Dec. 2014.

[23] M. Ji, G. Caire, and A. F. Molisch, "Fundamental limits of caching in wireless D2D networks," arXiv preprint arXiv:1405.5336, 2014.

[24] M. Ji, G. Caire, and A. F. Molisch, "The Throughput-Outage Tradeoff of Wireless One-Hop Caching Networks," arXiv preprint arXiv:1312.2637, 2013. 
[25] M. Ji, G. Caire, and A. F. Molisch, "Wireless Device-to-Device Caching Networks: Basic Principles abd System Performance," arXiv preprint arXiv:1305.5216, 2014.

[26] N. Golrezaei, P. Mansourifard, A. F. Molisch, and A. G. Dimakis, "Base-Station Assisted Device-to-Device Communications for High-Throughput Wireless Video Networks," IEEE Trans. Wireless Commun., vol. 13, no. 7, pp. 3665-3676, Jul. 2014.

[27] S. W. Jeon, S. N. Hong, M. Ji, and G. Caire, "Caching in wireless multihop device-to-device networks," IEEE International Communication Conference (ICC), 2015.

[28] S. W. Jeon, S. N. Hong, M. Ji, and G. Caire, "On the capacity of multihop device-to-device caching networks," IEEE Information Theory Workshop (ITW), 2015.

[29] M. Cha, H. Kwak, P. Rodriguez, Y. Ahn, and S. Moon, "I tube, you tube, everybody tube: Analyzing the world's largest user generated content video system," in Proc. 7th ACM SIGCOMM conf. Internet Meas., 2007, pp. 1-14.

[30] J. A. Bondy and U. S. R. Murty, "Graph theory with applications," vol. 290, London: Macmillan, 1976.

[31] R. S. Garfinkel and G. L. Nemhauser, “Interger Programming,” John Wiley \& Sons, New York, 1972.

[32] L. B. Le and E. Hossain, "Resource allocation for spectrum underlay in cognitive radio networks," IEEE Trans. Wireless Commun., vol. 7, no. 12, pp. 5306-5315, Dec. 2008.

[33] X. Wu, S. Tavildar, S. Shakkottai, T. Richardson, J. Li, R. Laroia, and A. Jovicic, "FlashLinQ: A synchronous distributed scheduler for peer-to-peer ad hoc networks," IEEE/ACM Transactions on Networking, vol. 21, no. 4, pp. 1215-1228, Aug. 2013.

[34] N. Naderializadeh and A. S. Avestimehr, "ITLinQ: A new approach for spectrum sharing in device-to-device communication systems," IEEE J. Select. Area Commun., vol. 32, no.6, pp. 1139-1151, Jun. 2014.

[35] S. Sarkar, P. Chaporkar, and K. Kar, "Fairness and Throughput Guarantees with Maximal Scheduling in Multi-hop Wireless Networks," IEEE Proceedings of WiOpt, 2006.

[36] S. Boyd and L. Vandenberge, Convex Optimization. Cambridge Unversity Press, 2004.

[37] D. Bernstein, "Matrix Mathematics," Princeton University Press., ISBN 0-691-11802-7.

[38] J. L. Bentley, "Multidimensional binary search trees in database applications," IEEE Trans. Software Engineering, vol. SE-5, no. 4, pp. 333-340, Jul. 1979.

[39] K. Doppler, C. H. Yu, C. B. Ribeiro, and P. Janis, "Mode Selection for Device-to-Device Communication underlaying an LTE-advanced Network," in Proc. IEEE Wireless Communications and Networking Conference (WCNC), 2010. 